\title{
MicroRNA-181a is elevated by 10-hydroxycamptothecin and represses lung carcinoma progression by downregulating FOXP1
}

\author{
Li PAN ${ }^{1}$; Wenting YI ${ }^{2}$; Dongmin LIANG ${ }^{1}$; Yulong ZHAO $^{1}$; RAnRan WANG ${ }^{1}$; Pingyu WANG ${ }^{1}$; Youjie LI $^{1}$; JiAXuan XIN ${ }^{1}$; \\ YUNFEI YAN ${ }^{1, *, *}$; SHUYANG XIE ${ }^{1, * * \#}$ \\ ${ }^{1}$ Department of Biochemistry and Molecular Biology, Binzhou Medical University, Yantai, 264003, China \\ 2 Department of Medical Laboratory, Yantai Affiliated Hospital of Binzhou Medical University, Yantai, 264100, China
}

Key words: microRNA-181a, 10-hydroxycamptothecin, Angiogenesis, Lung carcinoma

\begin{abstract}
Tumor progression is usually characterized by proliferation, migration, and angiogenesis, which is essential for supplying both nutrients and oxygen to the tumor cells. Therefore, targeting angiogenesis has been considered a promising therapeutic strategy for cancer prevention and treatment. In the present study, we demonstrated that in addition to suppressing lung cancer cell proliferation and migration in vitro, 10-hydroxycamptothecin (10-HCPT) is also capable of inhibiting angiogenesis in vivo with a miR-181a-dependent manner. Mechanistically, by upregulating miR-181a, which in turn downregulating FOXP1, 10-HCPT can inhibit the PI3K/Akt/ERK signaling pathwaymediated angiogenesis. Furthermore, reduced levels of miR-181a have been found in both lung cancer cell lines and xenograft with concurrently elevated levels of FOXP1, VEGF, bFGF, and HDGF. Consistent with the findings from the in vitro experiments, miR-181a impairs neovascularization in our xenograft model. In summary, our findings have not only established the anti-oncogenic role of miR-181a in lung cancer angiogenesis but also suggest that 10-HCPT could be a potential therapeutic reagent for lung cancer treatment.
\end{abstract}

$\begin{array}{ll}\text { Abbreviations } \\ \text { 10-HCPT: } & \text { 10-hydroxycamptothecin } \\ \text { bFGF: } & \text { basic fibroblast growth factor } \\ \text { ERK: } & \text { extracellular regulated protein kinases } \\ \text { FOXP1: } & \text { Forkhead box protein P1 } \\ \text { HDGF: } & \text { hepatoma-derived growth factor } \\ \text { MMP: } & \text { matrix metalloproteinase } \\ \text { PI3K: } & \text { phosphatidylinositol 3-kinase } \\ \text { VEGF: } & \text { vascular endothelial growth factor }\end{array}$

\section{Introduction}

Lung cancer is one of the most common malignant human cancers worldwide and shows characteristics features of uncontrolled cell growth, high invasion, and rapid metastasis (Cersosimo, 2002). The greatest indication for

\footnotetext{
*Address correspondence to: Shuyang Xie, shuyangxie@aliyun.com; Yunfei Yan, flycloud1982@163.com

${ }^{\#}$ These authors contributed equally to this work

Received: 05 January 2021; Accepted: 30 March 2021
}

lung cancer cure is confirmed diagnosis at the early stage. In 2014, the UK Office for National Statistics reported that patients diagnosed with distant metastatic disease (Stage IV) had a 1-year survival rate of just $15 \%-19 \%$ compared with 81\%-85\% for Stage I (Blandin Knight et al., 2017).

The progression of solid tumors is critically dependent on a functional vascular supply, in the absence of which tumors remain dormant and unable to metastasize. Several anticancer drugs such as 10-hydroxycamptothecin (10-HCPT) have been tested in clinical and basic studies as angiogenesis inhibitors, in addition to their demonstrated functions in suppressing cell proliferation and metastasis. 10-HCPT is a derivative of camptothecin, which was discovered as a plant antitumor agent and showed to specifically inhibit the DNA topoisomerase I to arrest the cell cycle and induce apoptosis (Wani et al., 1980; Ling et al., 1990).

Although 10-HCPT has been revealed as a potential antiangiogenic agent, the detailed underlying mechanisms still need to be elucidated (Xiao et al., 2001; Olgen, 2018).

Anti-angiogenic drugs function by suppressing proangiogenic factors, such as vascular endothelial growth factor (VEGF), bFGF (basic fibroblast growth factor), HDGF (hepatoma-derived growth factor), HIF-1a (hypoxia-inducible factor), MMP2, and MMP9 (matrix metalloproteinase), which 
are highly expressed in tumor cells. These angiogenic factors trigger a series of pro-angiogenic reactions, resulting in the proliferation and migration of endothelial cells. Although clinical trials have shown that anti-angiogenic agents notably improve the therapeutic index of anticancer drugs, a great challenge is to identify more effective anti-angiogenic targets (Vasudev and Reynolds, 2014; Gacche and Meshram, 2014; Bergers and Benjamin, 2003; Eguchi and Wakabayashi, 2020). Hence, elucidation of signaling pathways of anti-angiogenic targets is one of the solutions to challenges.

MicroRNAs (miRNAs), small and highly conserved noncoding RNAs, are involved in a wide array of cellular and molecular processes. Numerous studies have demonstrated that miRNAs play important roles in a range of processes, including cell proliferation, metastasis, and angiogenesis, which are key pathogenic events in the carcinoma progression (Yang and Yin, 2014; Zhou et al., 2017). The miR-181 family has been reported to possess highly conserved sequences in almost all vertebrates and is involved in virtually all major signaling pathways via binding the 3 ' untranslated region (UTR) of multiple targeted mRNAs (Rezaei et al., 2020). Furthermore, many studies have demonstrated that the miR-181 family members are dysregulated in various tumor tissues and play important roles in carcinogenesis (Ji et al., 2009). Several reports have also shown that members of the miR-181 family serve as oncogenes or tumor suppressors, even in one type of cancer. For example, miR-181a both promotes proliferation and suppresses migration in colon cancer cells (Wei et al., 2014; Li et al., 2015).

In the present study, we demonstrated that miR-181a expression was attenuated in both lung carcinoma cell lines and lung carcinoma tissues. Functional studies revealed that miR181a played an anti-oncogenic role in suppressing proliferation and migration of lung carcinoma cells. Additionally, miR-181a transfection could reduce the expression of pro-angiogenic factors in lung carcinoma cells. Consistently, neovascularization in xenografts was also suppressed by miR-181a transfection. The underlying mechanism may involve the downregulation of Forkhead box protein P1 (FOXP1) expression by miR-181a and the consequently decreased expression of pro-angiogenic factors to modify the tumor environment. We also found that 10 HCPT, which elevated miR-181a expression, repressed proliferation, migration, and angiogenesis. Together, our findings demonstrate that miR-181a plays a negative role in cancer dissemination and may serve as a potential prognostic biomarker and molecular target for anti-angiogenic drug development.

\section{Materials and Methods}

\section{Cell culture and reagents}

The cell lines (A549, NCl-H1975, H1299, and HBE) were provided by the Institute of Shanghai Cell Biology. A549, H1975, H1299, and HBE cells were kept in RPMI-1640 medium (Sigma-Aldrich; Merck KGaA) supplemented with $10 \%$ fetal bovine serum (Hyclone; GE Healthcare). The following drugs and reagents were used in the present study: 3-(4,5-dimethylthiazol-2-yl)-2,5-diphenyltetrazolium bromide (MTT; Sigma-Aldrich; Merck KGaA), trypsin (Sigma-Aldrich; Merck KGaA), DMSO (Sigma-Aldrich; Merck KGaA),
$5 \times$ poly-A buffer, $\mathrm{MgCl}_{2}$, and dATP (Promega Corporation), dNTP mixture and RNase inhibitor (Takara Bio, Inc., Shiga, Japan), SYBR Green PCR master mix quantification PCR kit (Qiagen, Inc., Hilden, Germany), Matrigel (E6909, SigmaAldrich; Merck KGaA), VEGF (AmyJet Scientific; Wuhan; China), 10-Hydroxycamptothecin (Solarbio, Inc., Metro Manila, the Philippines).

\section{Transfection}

$1 \times 10^{6}$ A549 cells were transfected with $1 \mu \mathrm{g}$ siRNA (or plasmids) in $2.5 \mu \mathrm{L}$ of Lipofectamine 2000 (Invitrogen, Carlsbad, CA, USA) according to the manufacturer's instructions. All transfections were carried out in triplicate.

\section{MTT assay}

A549 cells were seeded at 5000 cells/well in 96-well plates. Transfection or chemical dosing was conducted in a well after $24 \mathrm{~h}$ incubation. After incubation for $48 \mathrm{~h}, 10 \mu \mathrm{L}$ of $5 \mathrm{mg} / \mathrm{mL}$ MTT were added into each well before the end of the experiment and then incubated for $4 \mathrm{~h}$ at $37^{\circ} \mathrm{C}$. The culture medium was discarded, and $100 \mu \mathrm{L}$ of DMSO was added to each well; the plate was shaken for $10 \mathrm{~min}$ at room temperature. Absorbance (OD) value was measured at $570 \mathrm{~nm}$ with a Microplate Reader (Multiskan FC; Thermo Fisher Scientific, Inc., Waltham, USA). Each experiment was repeated a minimum of three times.

\section{Cell migration assay}

The migration ability of A549 cells was assessed using transwell chambers in the present study. Firstly, A549 cells were harvested and resuspended in a serum-free medium. Then cells $\left(1 \times 10^{4}\right.$ cells/well $)$ were directly added to the upper chamber of the transwell culture plates. Medium containing $20 \%$ FBS was added to the lower chamber of the transwell culture plates to serve as a chemoattractant. After incubation for $24 \mathrm{~h}$ in an incubator at $37^{\circ} \mathrm{C}$ and $5 \% \mathrm{CO}_{2}$ condition, non-migrating cells adhered on the upper surface of the membrane were scrubbed gently with a cotton-tipped swab. Then cells migrating to the lower surface of the membrane were fixed with $4 \%$ paraformaldehyde for $30 \mathrm{~min}$, stained with $10 \%$ crystal violet for $15 \mathrm{~min}$, and washed with PBS. Finally, cells in five randomly selected fields of each chamber were photographed with an inverted microscope and quantified by manual counting and used to indirectly reflect the tumor cell migration ability.

\section{Cell apoptosis assay}

For apoptosis analysis by flow cytometry, A549 Cells $\left(4 \times 10^{5}\right.$ cells/well) were seeded in 6-well plates and incubated overnight, then treated with 10-hydroxycamptothecin or miR-181a for $48 \mathrm{~h}$. The cells were harvested with trypsin and washed with PBS, stained with Annexin V-FITC/PI kit (Nanjing KeyGen Biotech Co., Ltd., Nanjing, China) according to the manufacturer's protocols. Finally, the samples were analyzed by BD FACS Diva Software on a flow cytometer (BD FACS Canto TMII, BD Bioscience).

\section{Colony formation assay}

The processed cell suspension was collected, and 1000 cells were seeded in a $100 \mathrm{~mm}$-culture dish and cultured in a cell 
culture incubator for 2 weeks. When visible clones were observed, the cell colonies were washed three times with PBS. Colonies were fixed with $4 \%$ paraformaldehyde for $30 \mathrm{~min}$ and stained with $0.1 \%$ crystal violet for $20 \mathrm{~min}$. Finally, the number of clones was counted in each culture dish.

\section{Western blotting}

The cells were lysed in RIPA buffer (Beyotime Institute of Biotechnology) containing protease inhibitor cocktail at $4^{\circ} \mathrm{C}$ on ice for $30 \mathrm{~min}$. After centrifugation at $12,000 \mathrm{rpm}$ for $10 \mathrm{~min}$ at $4^{\circ} \mathrm{C}$, equal amounts of protein samples $(30 \mu \mathrm{g})$ were prepared for electrophoresis running on $10 \%$ sodium dodecyl sulfate polyacrylamide gel electrophoresis (SDS-PAGE) and transferred onto a polyvinylidene difluoride (PVDF) membrane. The membranes were then blocked by incubating with blocking buffer (5\% fat-free milk/TBST, $\mathrm{pH}$ 7.4) for $1 \mathrm{~h}$ at room temperature. The membranes were probed with primary antibodies as follows: rabbit anti-GAPDH (AP0063, Bioworld Technology, Inc., St Louis, USA), rabbit anti-AKT (BS4007, Bioworld Technology, Inc., St Louis, USA), rabbit anti-phosphoAKT (bs-0876R, Bioss Inc., Woburn, USA), rabbit anti-MMP9 (BS6893, Bioworld Technology, Inc., St Louis, USA), rabbit antiHIF1- $\alpha$ (BS3514, Bioworld Technology, Inc., St Louis, USA), rabbit anti-Bax (BS2538, Bioworld Technology, Inc., St Louis, USA), rabbit anti-Bcl-2 (BS70205, Bioworld Technology, Inc., St Louis, USA), rabbit anti-ERK (bs-0022R, Bioss Inc., Woburn, USA), rabbit anti-phospho-ERK (bsm-33353M, Bioss Inc., Woburn, USA), Rabbit anti-PI3K (bs-0128R, Bioss Inc., Woburn, USA), rabbit anti-phospho-PI3K (abs130868, Absin), rabbit anti-FOXP1 (ab134055, Abcam) were incubated overnight at $4^{\circ} \mathrm{C}$. Membranes were washed with TBST three times, and then horseradish peroxidase (HRP) conjugated secondary antibodies (BS13278, Bioworld Technology, Inc., St Louis, USA) were incubated for $1 \mathrm{~h}$ at room temperature. Finally, signals were captured using enhanced chemiluminescence (ECL, Wuhan Boster Biological Technology, Ltd., Wuhan, China). Densitometry was performed using a Gel Image System 4.2 (Tanon Science \& Technology Co, Ltd., Shanghai, China). All the western blot results were repeated three times.

\section{Quantitative RT-PCR ( $q R T-P C R)$ analysis}

Total RNA was isolated from the cells using the TRIzol reagent (Thermo Fisher Scientific, Inc., Waltham, USA), miRNAs were extracted from the cells using mirVana ${ }^{\mathrm{TM}}$ miRNA kit (Ambion; Thermo Fisher Scientific, Inc., Waltham, USA), and poly (A) was added using poly (A) polymerase, following the instruction of the manufacturer. Extracted RNA was reverse transcribed into cDNA with a Reverse Transcription Kit (Takara). qRT-PCR was performed to detect RNA levels using TB Green ${ }^{\circledR}$ Premix Ex Taq ${ }^{\mathrm{TM}}$ kit (RR420A, Takara Bio Company. Shiga, Japan). Data analysis was performed using the $2^{-\Delta \Delta \mathrm{Ct}}$ method. The primers sequences used for qRT-PCR were as follows: miR-181a forward: 5' AGGTTGCTAGGACATCAACGG 3'.

miR-181a reverse: 5’ AGAGTTAGTTTGGTAGCTGGC 3'. 5S rRNA forward: 5' GCCATACCACCCTGAACG 3'. 5S rRNA reverse: 5’ AACATGTACAGTCCATGGATG 3'. GAPDH forward: 5' GTCTTCACCACCATGGAGAAGG 3'.
GAPDH reverse: 5' GCCTGCTTCACCACCTTCTTGA 3'. VEGF forward: 5' AGGGCAGAATCATGAGCAAGT 3' VEGF reverse: 5' AGGGTCTGCATTGGATGGCA 3'. bFGF forward: 5' AGTGTCTGCTAACCGTTAGCT 3' bFGF reverse: 5' ACTGCCCAGTTCGTTTCAGTG 3'. HDGF forward: 5' ATCAACAGCCAACAAATACC 3' HDGF reverse: 5' TTCTTATCACCGTCACCCT 3' (Ke et al., 2013).

MMP2 forward: 5' GCTGATGTCCAGCGAGTG 3' MMP2 reverse: 5' TGCAGCCTAGCCAGTCG 3'.

\section{GFP report assays}

Plasmid GFP-FOXP1-3'UTR was constructed based on GV144 purchased from GENECHEM Co, Ltd. (Shanghai, China). The cloning sequence was part of FOXP1-3'UTR containing the possible binding sites of miR-181a. GFPFOXP1-3'UTR and siRNAs were co-transfected into A549 cells. After $24 \mathrm{~h}$, the GFP positive cells were observed and analyzed by FACS (Beckman) (Zhang et al., 2016).

The cloning sequence was as follows:

TTAAGTTCTGACCTCAGGCCTCCATTTGGGCCGATGGCCTCTTGGAGGCTTAAAGTTTTCTGTACCTTGTGATGAATGTTAATAGGTGTTTTTATTATACAAAGCTGAATGTCATTTCTCGTTTGTAGCTTTCTGTCACTCATTCCATCTTCCTTCAGACATCACCACGTTTCTCT-AAAG.

\section{Xenograft mouse model}

Six-week-old BALB/c-nu male mice were purchased from Nanjing GemPharmatech Co., Ltd., Nanjing, China. The mice were kept under sterile conditions. Each mouse was subcutaneously injected with A549 cell suspensions $\left(5 \times 10^{6}\right.$ cells) into the upper back of the mice. The experimental group was treated with $10-\mathrm{HCPT}(2 \mathrm{mg} / \mathrm{kg})$ by intraperitoneal injection, while the control group was treated with an equal volume of DMSO. Control cells and cells treated with miR-181a were subcutaneously tumorized of the upper back of the mice, respectively. Every three days, a vernier caliper was used to measure the tumor width and height, and the following formula was used to calculate the tumor volume: tumor volume = (length $\times$ width $\left.^{2}\right) / 2$. All mice were sacrificed after 4 weeks, and tumors were collected and weighed (Zhang et al., 2016). All animal experiments were approved by the Ethics Committee of Animal Experiments of Binzhou Medical University.

\section{Evaluation of xenograft angiogenesis}

Xenografts were fixed with $4 \%$ paraformaldehyde and embedded in paraffin. Immunohistochemistry was performed on $3 \mu \mathrm{m}$ sections to visualize vascular endothelium by using CD31 antibody (Cell signaling technology), which could only react with mice antigen. Three field images were collected from each slide by using an EVOS ${ }^{\mathrm{TM}}$ M7000 Imaging System (Thermo Fisher Scientific, Inc., Waltham, USA). The discrete cluster cells stained CD31 positive was counted down as one vessel. Tumor vessel density was reported as the average number of vessels number per field.

\section{Statistical analysis}

SPSS 22.0 software was used to analyze the data. All experiments were repeated three times, and the data were presented as the mean \pm SD. Statistical analysis between two 
groups was evaluated by unpaired or paired Student's $t$-test. ANOVA was used for the comparison of means of multiple groups. $P<0.05$ was considered to indicate a statistically significant difference.

\section{Results}

10-HCPT suppressed proliferation and metastasis but promoted apoptosis of lung cancer cells

We first treated A549 lung carcinoma cells with 10-HCPT at various concentrations for $48 \mathrm{~h}$ and performed MTT assays to evaluate cell proliferation. The results indicated that $10-\mathrm{HCPT}$ at concentrations from 0.5 to $2 \mu \mathrm{M}$ significantly suppressed cell proliferation (Fig. 1A). The half-maximal inhibitory concentration (IC50) value for 10-HCPT in A549 cells was 1 $\mu \mathrm{M}$, which was applied to the following experiments. As a result, 10-HCPT treatment remarkably impaired colony growth in colony formation assays compared with the DMSO control (Fig. 1B). We next evaluated the effects of 10-HCPT on cell apoptosis using flow cytometry. The results showed that apoptosis of A549 cells was significantly elevated by 10-HCPT treatment. In addition, the $10-\mathrm{HCPT}$ treatment decreased the expression of $\mathrm{Bcl} 2$ and increased the expression of $\mathrm{Bax}$ compared with the DMSO control (Fig. 1C). Moreover, Transwell migration assays demonstrated that 10-HCPT treatment notably attenuated A549 cell migration compared with the DMSO control (Fig. 1D).

Previous studies reported that FOXP1 and the related PI3K/Akt/ERK1/2 pathways play an important role in tumor progression (He et al., 2018). We performed western blot analysis to determine whether 10-HCPT had an impact on FOXP1 expression and the PI3K/Akt/ERK1/2 pathways. The results showed that $10-\mathrm{HCPT}$ attenuated the expression of FOXP1 and phosphorylation of PI3K, Akt, and ERK. In contrast, 10-HCPT treatment almost had no impact on the expression of total PI3K, Akt, and ERK (Fig. 1E).

To further investigate the anti-tumor function of 10-HCPT in vivo, A549 cells were injected into nude mice. After 12 days, 10 -HCPT or DMSO was intraperitoneally injected into nude mice. Every three days, the tumor volumes were measured. One month after A549 cell injection, the mice were sacrificed, and the tumor volumes and weights were measured. The results showed that 10-HCPT treatment suppressed tumor growth both in terms of volume and weight compared with the DMSO control (Fig. 1F). Angiogenesis in xenografts was evaluated by CD31 immunohistochemical staining. The number and density of novel blood vessels were decreased in xenografts treated with 10-HCPT compared with those treated with DMSO (Fig. 1G). We also conducted CD31 fluorescent staining to assess neovascularization in xenografts and obtained similar results (Suppl. Fig. S1). In addition, tumors with 10-HCPT treatment showed reduced expression of pro-angiogenic factors (VEGF, bFGF, HDGF, and MMP2) (Fig. 1H). These data indicated that 10-HCPT repressed suppressing proliferation, metastasis, and angiogenesis to restrain carcinoma progression.

Expression of miR-181a was elevated by 10-HCPT and upregulated in lung carcinoma tissue

Our results showed that 10-HCPT remarkably restrained the progression of lung carcinoma. We therefore next investigated the mechanism by which 10-HCPT exerts its anti-tumor effects in lung carcinoma. Previous studies reported that $10-\mathrm{HCPT}$ is involved in the regulation of several miRNAs (Zeng et al., 2019; Wu et al., 2011). miR181a has been uncovered involving into the anticancer process of several chemotherapy drugs (Zhao et al., 2016; Armstrong et al., 2017). In the present study, we found that the expression of miR-181a was notably upregulated in 10-HCPT-treated A549 cells (Fig. 2A). Furthermore, carcinoma tissue lysates prepared from 10 patients with lung cancer and the corresponding para-carcinoma tissues were analyzed for miR-181a expression. The expression of miR-181a in lung carcinoma samples was significantly decreased compared with levels in the corresponding para-carcinoma tissues (Fig. 2B). We also analyzed miR-181a expression in several cell lines and found that miR-181a levels were reduced in lung carcinoma cell lines (A549, H1975, and H1299) compared with levels in the human bronchial epithelial (HBE) cell line (Fig. 2C). Moreover, we also detected the expression of several proangiogenic factors (VEGF, bFGF, HDGF, and MMP2), which were found elevated in lung carcinoma cell lines (A549, H1975, and H1299) compared with HBE (Suppl. Fig. S1B). These data indicated that miR-181a may play an anti-oncogenic role in lung carcinoma.

miR-181a suppressed proliferation and migration of cancer cells and promoted apoptosis in addition to downregulating the expression of pro-angiogenic factors

To further investigate the anti-oncogenic function of miR181a in lung carcinoma, we prepared miR-181a (mimic) and miR-181a inhibitor (antisense oligonucleotide) along with their respective controls for transfection of A549 cells. We confirmed that miR-181a mimic transfection of A549 cells resulted in significantly elevated miR-181a levels, while levels were reduced upon transfection with miR-181a inhibitor (Fig. 3A). MTT assay was conducted to assess A549 cell proliferation. The results indicated that miR-181a transfection significantly suppressed the proliferation of A549 cells (Fig. 3B), while miR-181a inhibitor transfection resulted in promoting A549 cell proliferation (Fig. 3C). Furthermore, colony formation assays indicated that miR181a suppressed cell survival of A549 seeded sparsely in a clonogenic assay compared with the control (Fig. 3D). Conversely, cells transfected with the miR-181a inhibitor formed more colonies compared with the scrambled control (Fig. 3E). Flow cytometry showed that miR-181a transfection induced more A549 cell apoptosis compared with the control (Fig. 3F). Consistent with these findings, miR-181a transfection downregulated the expression of $\mathrm{Bcl} 2$ and upregulated Bax expression (Fig. 3F). Although apoptosis was not significantly attenuated by miR-181a inhibitor transfection, elevated expression of $\mathrm{Bcl} 2$ and decreased expression of Bax were observed with miR-181a inhibitor transfection (Fig. 3G).

We also assessed the role of miR-181a in cell migration using Transwell migration assays, which showed that A549 cells transfected with miR-181a had decreased migration capacity compared with cells transfected with the control (Fig. $3 \mathrm{H}$ ). In contrast, miR-181a inhibitor transfection elevated cell migration compared with the scrambled control (Fig. 3I). 
A

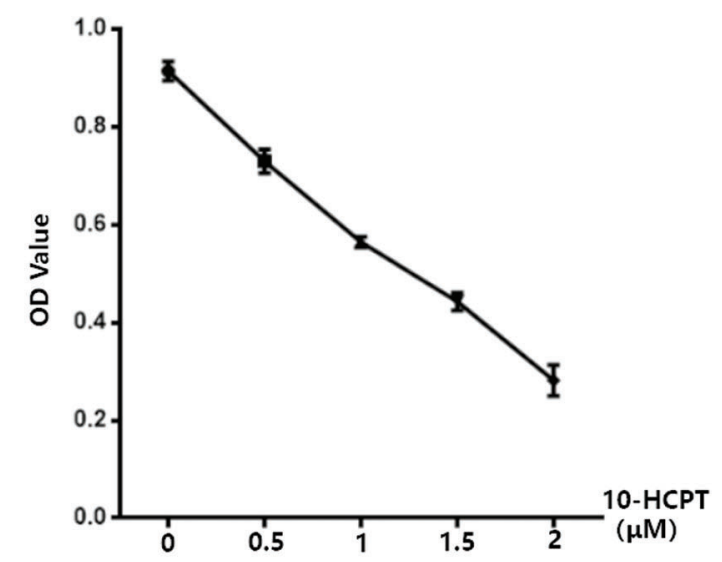

C

E

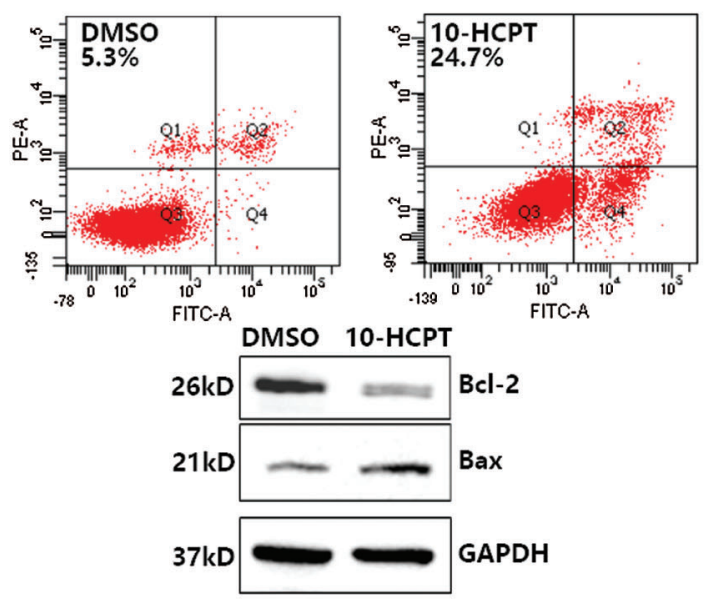

B
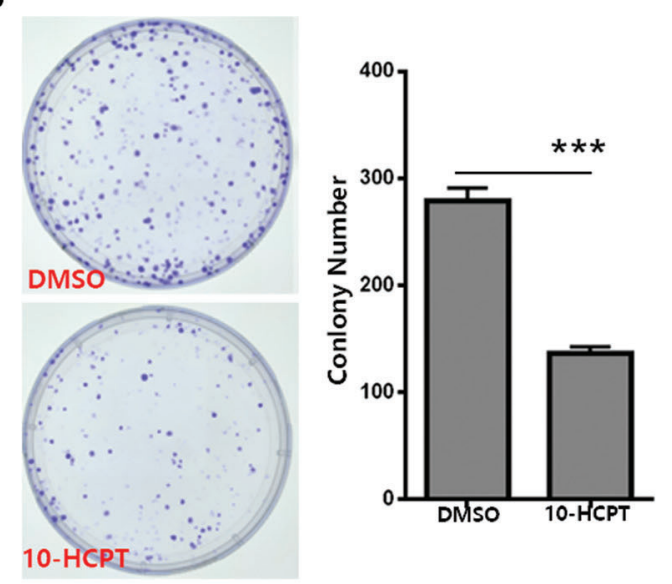

D

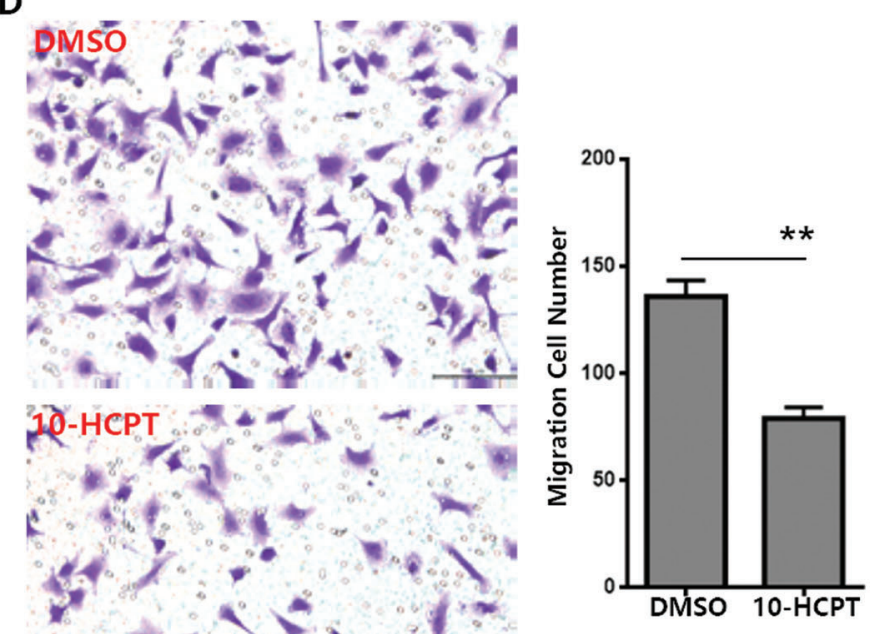

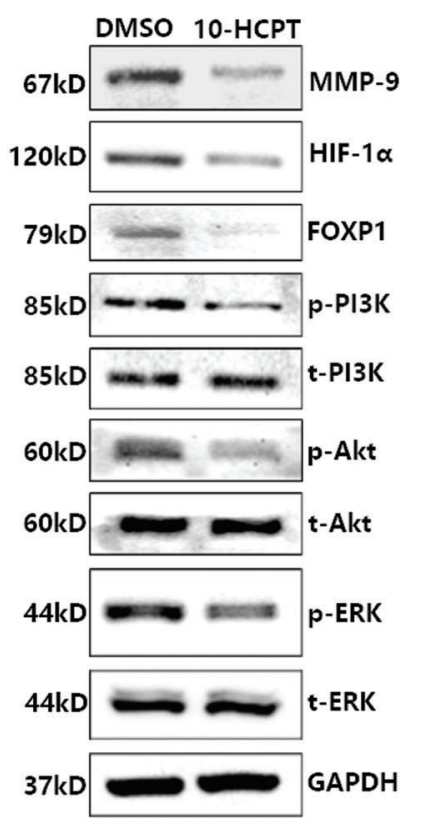

$\mathbf{F}$
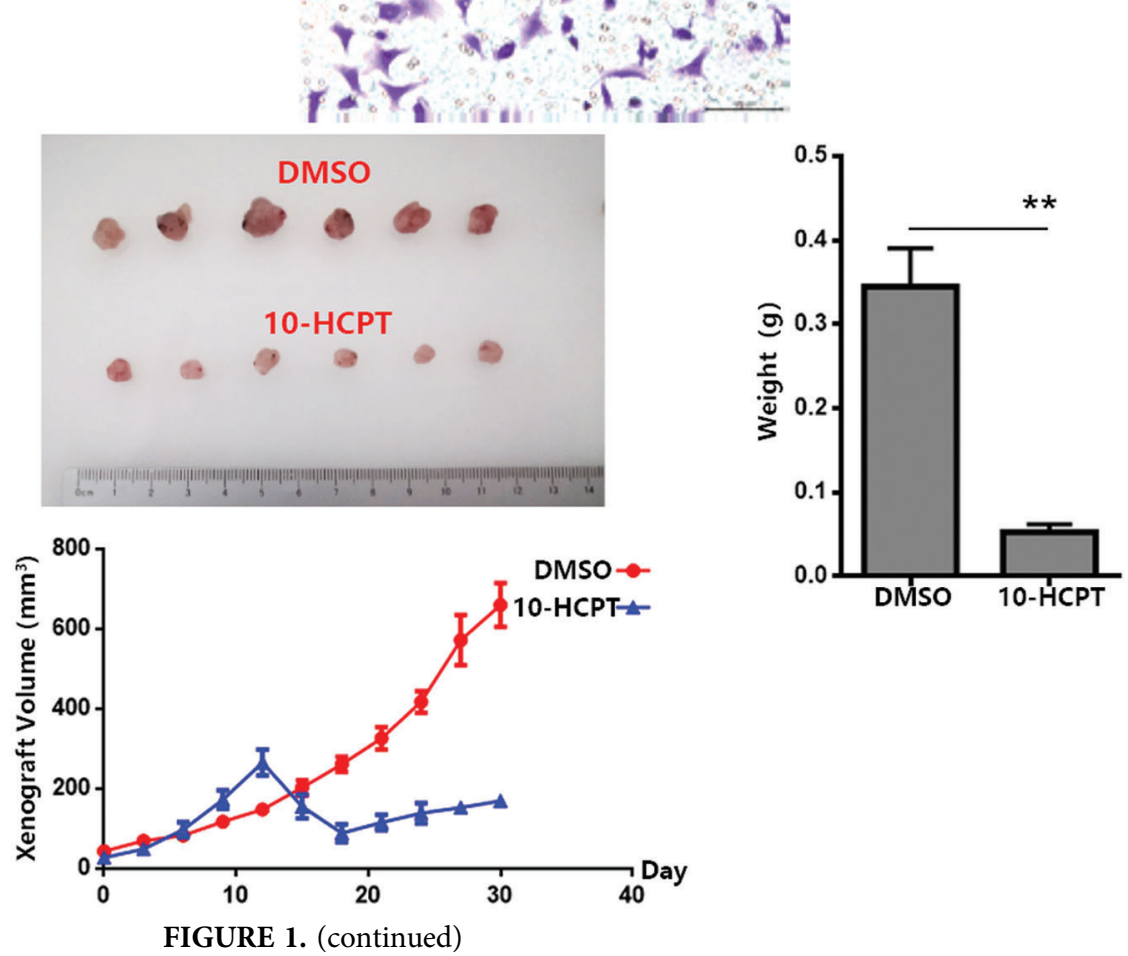
G

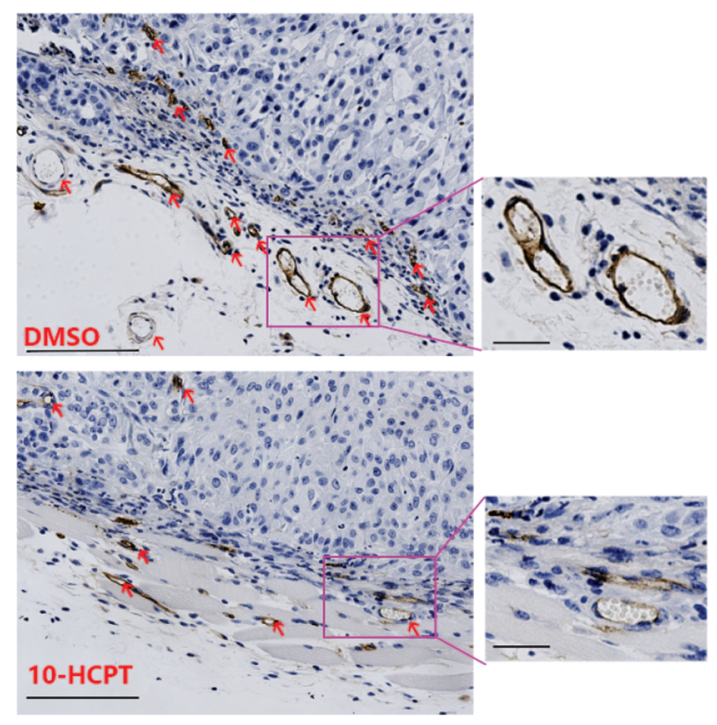

H

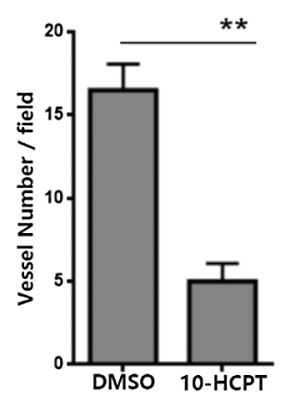

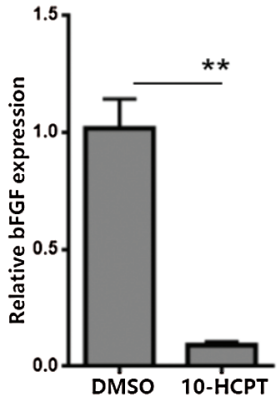
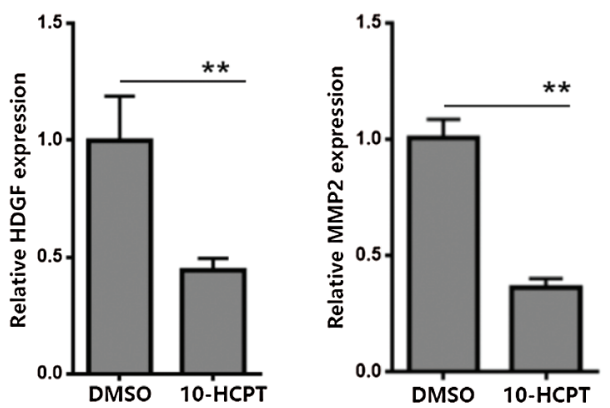

FIGURE 1. 10-Hydroxycamptothecin suppressed proliferation, metastasis, and angiogenesis but promoted apoptosis of lung cancer cells. (A) The proliferation curve of A549 cells treated with 10-HCPT in different concentrations for $48 \mathrm{~h}$. Data are expressed as mean \pm SD for triplicate experiments. (B) Colony formation assays of A549 cells treated with 10-HCPT or DMSO for $48 \mathrm{~h}$. Data are expressed as mean \pm SD for triplicate experiments. ${ }^{* *} P<0.001$; unpaired $t$-test. (C) FACS and western blot analysis of A549 cell apoptosis at $48 \mathrm{~h}$ posttreatment of 10-HCPT and DMSO. upper panel: FACS analysis of A549 cells; lower panel: western blot analysis of Bcl2 and Bax expression. (D) Transwell migration assay of A549 at $48 \mathrm{~h}$ post-treatment of 10-HCPT or DMSO. Data are expressed as mean \pm SD for triplicate experiments. ${ }^{* * P}<0.01$; unpaired $t$-test. (E) Western blot detection of FOXP1 expression and activation of PI3K/Akt/ERK pathway in lung carcinoma cells treated with 10-HCPT or DMSO. (F) Analysis of xenograft formed by A549 cells treated with 10-HCPT or DMSO $(\mathrm{N}=6)$. Quantitative analysis of tumor volume (lower panel) and tumor weight (right panel) of xenografts. Data are expressed as mean $\pm \mathrm{SD}$. ${ }^{*} P<0.05$; Mann-Whitney U-test. $(\mathrm{G})$ Representative images of $\mathrm{CD} 31$ immunohistochemical staining of xenograft treated with 10-HCPT or DMSO. The red arrows indicated novel blood vessels formed by endothelial cells in xenografts. The neovascularization part was magnified. Blood vessels were quantified on one field from each xenograft. Data are expressed as mean \pm SD for triplicated fields. ${ }^{*} P<0.01$; unpaired $t$-test. (H) qRT-PCR analysis of VEGF, bFGF, HDGF, and MMP2 expression in A549 cells treated with 10-HCPT or DMSO. Data are expressed as mean \pm SD for triplicate experiments. ${ }^{* *} P<0.01$; unpaired $t$-test.

To elucidate the role of miR-181a in regulating the expression of pro-angiogenic factors, we performed the qRTPCR to analyze the expressions of VEGF, bFGF, HDGF, and MMP2, which were all suppressed as a result of miR-181a transfection in A549 cells (Fig. 3J). Western blot revealed that cells transfected with miR-181a had decreased MMP9 and HIF-1a expression compared with control cells (Fig. 3K). In contrast, the expression of pro-angiogenic factors, VEGF, bFGF, HDGF, MMP2, MMP9, and HIF-1 $\alpha$, were all elevated in A549 cells transfected with miR-181a inhibitor compared with cells transfected with the control (Figs. 3L and 3M). These data indicated that miR-181a played an anti-oncogenic role in suppressing proliferation, migration, expression of angiogenic factors, and promotion of apoptosis.

\section{miR-181a targeted the 3'UTR of FOXP1, which was elevated in lung carcinoma tissue}

To examine the mechanism underlying the anti-angiogenic role of miR-181a, we used miRNA TargetScan (http://www. targetscan.org/vert_72/) to predict the mRNAs that may bind to miR-181a. The results revealed that FOXP1, which functions in the regulation of vascular function, might be a target of miR-181a (Fig. 4A). We constructed a GFP reporter plasmid containing the FOXP1-3'UTR, which was used to co-transfect A549 cells together with miR-181a. miR-181a transfection attenuated both the percentage of GFP-positive cells (Fig. 4B) and GFP intensity (Fig. 4C). In addition, western blot revealed that FOXP1 expression was downregulated by miR-181a transfection and upregulated by miR-181a inhibitor transfection (Fig. 4D). We then examined the activation of PI3K/Akt pathways, which are enhanced by FOXP1 and play an important role in tumor angiogenesis. miR-181a transfection decreased the phosphorylation of PI3K, Akt, and ERK, while miR-181a inhibitor transfection resulted in increased PI3K, Akt, and ERK phosphorylation (Fig. 4D).

To examine the expression of FOXP1 in lung cancer cases, we collected carcinoma tissues and the corresponding para-carcinoma tissues from 12 patients diagnosed with lung cancer. Consistent with the miR-181a attenuation in lung carcinoma tissues, the expression of FOXP1 was significantly elevated in lung carcinoma tissues (Fig. 4E). These data suggested that the anti-angiogenic function of miR-181a may be through FOXP1/PI3K/Akt pathways.

miR-181a suppressed tumorigenesis and angiogenesis in a xenograft model

To investigate the effects of miR-181a on tumor growth in vivo, we constructed a xenograft model by injecting A549 
A

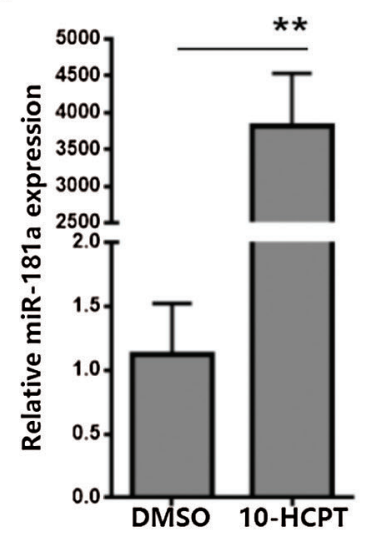

B

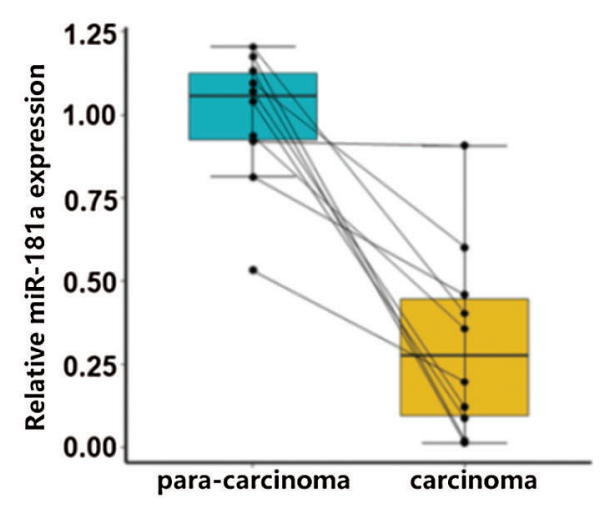

C

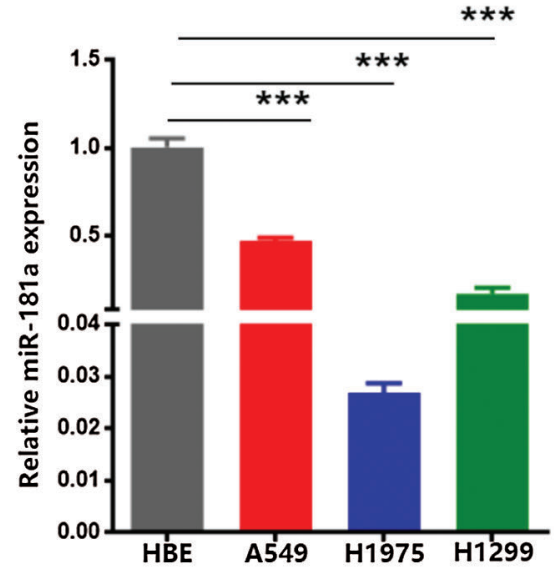

FIGURE 2. Expression of miR-181a elevated by 10-HCPT was upregulated in lung carcinoma.

(A) qRT-PCR analysis of miR-181a expression in A549 cells treated with 10-HCPT or DMSO. Data are expressed as mean \pm SD for triplicate experiments. ${ }^{*} P<0.01$; unpaired $t$-test. (B) qRT-PCR analysis of miR-181a expression in lung carcinoma tissue and the corresponding paracarcinoma tissue. Data are expressed as mean \pm SD for triplicate experiments. ${ }^{\star *} P<0.001$; paired $t$-test. (C) qRT-PCR analysis of miR-181a expression in A549, H1975, H1299, and HBE cells. Data are expressed as mean \pm SD for triplicate experiments. ${ }^{* * *} P<0.01$; ANOVA test.

cells transfected with miRNAs into nude mice. After injection, xenograft tumors were measured every 3 days. At the endpoint of one month, mice were sacrificed, and tumor tissues were harvested, photographed, and examined. The tumors formed from cells transfected with miR-181a showed decreased tumor volume and weight compared with the controls (Fig. 5A). In contrast, miR-181a inhibitor transfection resulted in enhanced tumor volume and weight compared with the scrambled control (Fig. 5B).

We further assessed angiogenesis in the xenografts and found that the novel vessel number was decreased in xenografts formed by A549 cells with miR-181a transfection compared with the controls (Fig. 5C). In contrast, miR-181a inhibitor transfection resulted in increased novel vessel formation in xenografts compared with the scrambled control (Fig. 5D). We obtained similar results with CD31 fluorescent staining (Suppl. Fig. S1C-S1D). We analyzed the expression of pro-angiogenic factors (VEGF, bFGF, HDGF, and MMP2) in xenografts by qRT-PCR and found that all factors were decreased in xenografts formed by A549 cells with miR-181a transfection compared with the control (Fig. 5E). In contrast, miR-181a inhibitor transfection resulted in increased VEGF, bFGF, HDGF, and MMP2 expression in xenografts (Fig. 5F).

The xenograft formed by A549 cells transfected with miR-181a showed decreased FOXP1 expression compared with the control. In contrast, FOXP1 expression was elevated in xenografts derived from cells transfected with the miR-181a inhibitor compared with controls (Fig. 5G). These data revealed that miR-181a suppressed the growth of xenograft in which neovascularization was also decreased.

\section{Discussion}

Tumor progression results from a series of complex and multifaceted interactions between tumor cells and their microenvironment. During tumor progression, a number of angiogenic factors are secreted in the tumor microenvironment and recruit vascular endothelial cells to form the intratumoral capillaries (Viallard and Larrivee, 2017; Carmeliet and Jain, 2011). Anti-angiogenic therapies have been developed and applied in the clinic. However, approaches aimed at targeting tumor neoangiogenesis have been proven successful only if the higher order of complexity of the tumor microenvironment is considered (Rahma and Hodi, 2019). Many reports revealed that miRNAs are involved in the regulation of angiogenic factors to modify the tumor microenvironment (Rupaimoole et al., 2016). In the present study, we found that miR-181a significantly suppressed cell proliferation and migration but promoted apoptosis of lung carcinoma cells. Moreover, miR-181a transfection not only attenuated the growth of xenografts but also decreased novel vessel formation in the xenografts.

Several studies have demonstrated that the function of miR-181a is tumor-specific. In ovarian, liver, and breast cancers, miR-181a enhanced cancer progression by different mechanisms. However, miR-181a plays tumor-suppressive roles in leukemia, glioma, and oral squamous cell carcinoma. In the present study, we found that the level of miR-181a was significantly decreased in lung carcinoma tissues and cell lines. Moreover, miR-181a was elevated in lung carcinoma cells treated by 10-HCPT, which is a chemotherapy drug, and suppressed the angiogenesis of endothelium cells by attenuating the expression of angiogenic factors in lung carcinoma cells. Many chemotherapy drugs are not only toxic to carcinoma cells but also modify the tumor microenvironment (Gao et al., 2014). miR-181a may play such a role in regulating the lung carcinoma microenvironment. Our results indicated that miR181a attenuated the expressions of VEGF, bFGF, HDGF, MMP2, MMP9, and HIF-1a in A549 cells. In non-small cell lung cancer, HDGF enhances VEGF-dependent angiogenesis, in which bFGF-2 is a VEGF-independent angiogenic factor (Eguchi and Wakabayashi, 2020). VEGF and bFGF are regulated by HIF-1a and not only stimulate the increase of vascular permeability but also trigger the migration of endothelial cells (Bradshaw et al., 2013; Chen et al., 2018). MMP2 and MMP9 are associated with degradation of the extracellular matrix and the advancement of tumor metastasis 
A

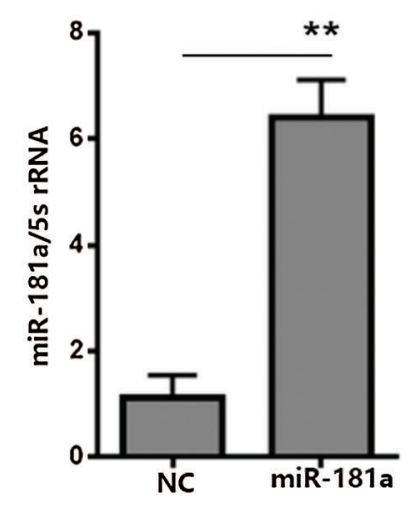

D

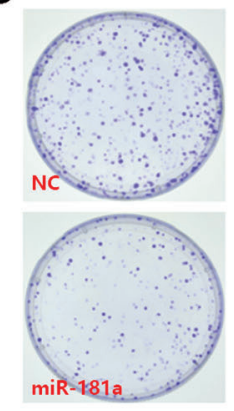

F
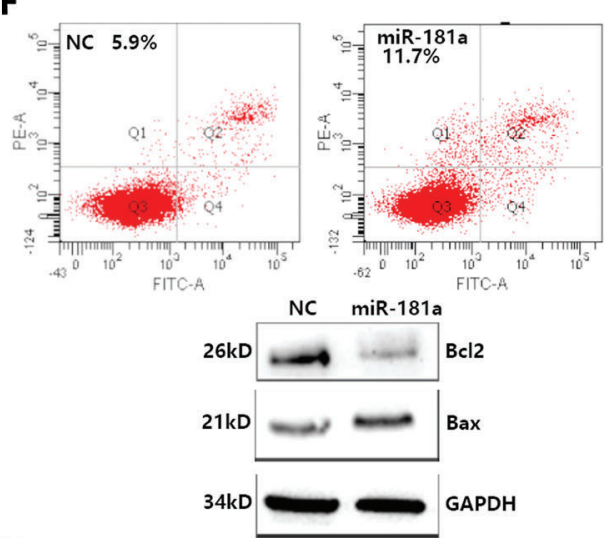

H
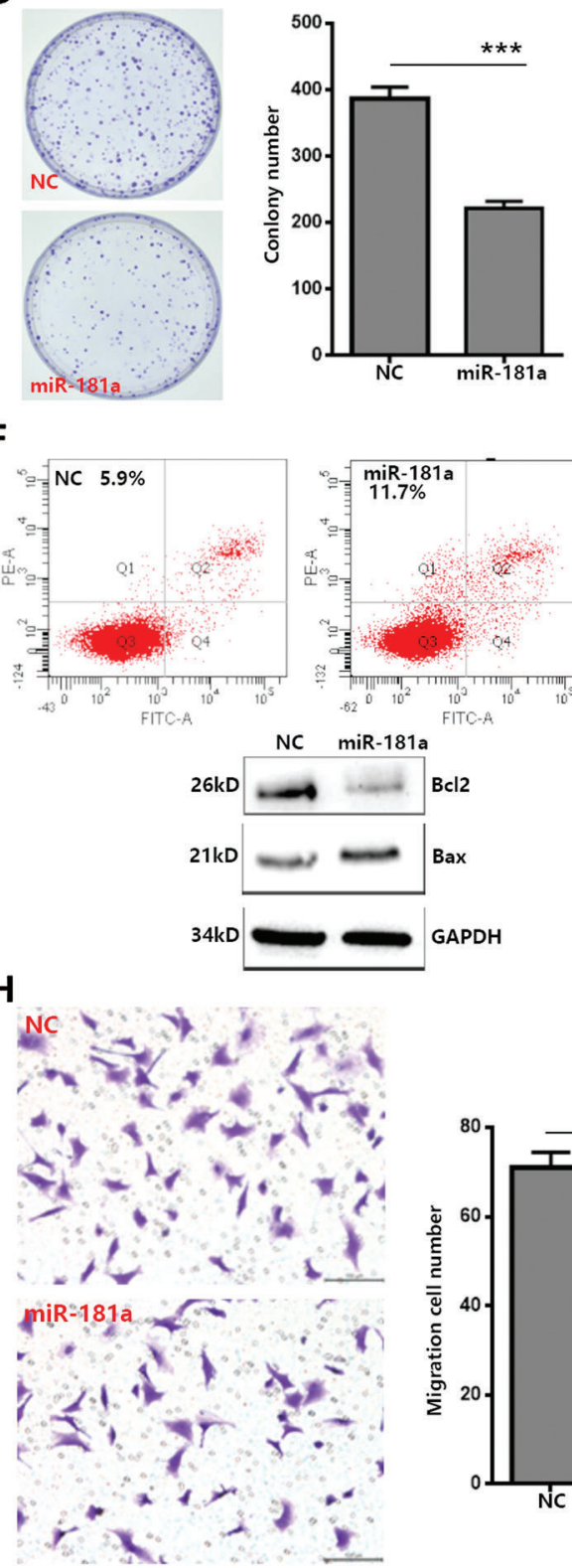

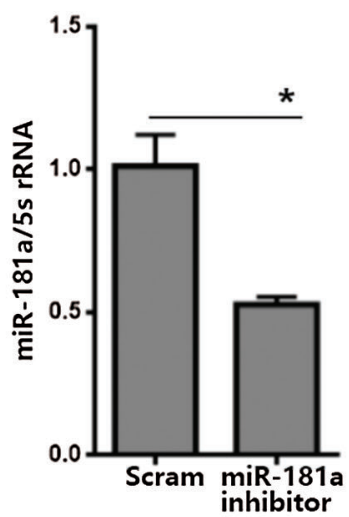

B

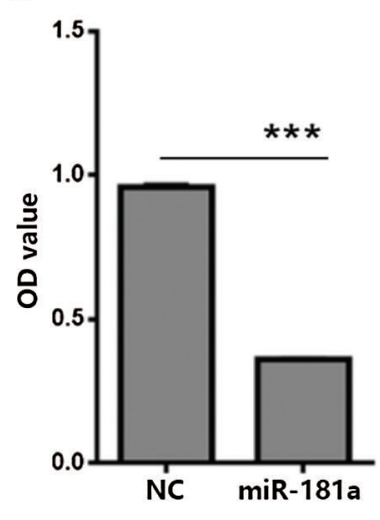

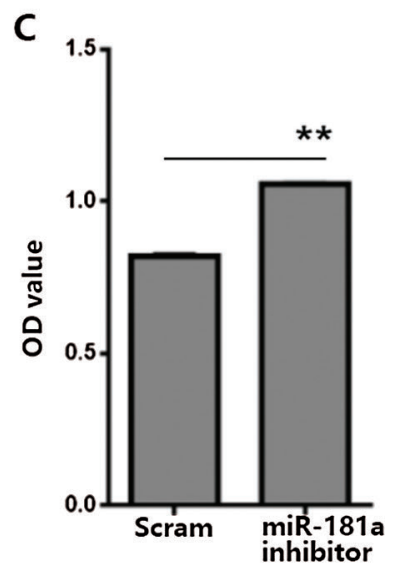

E
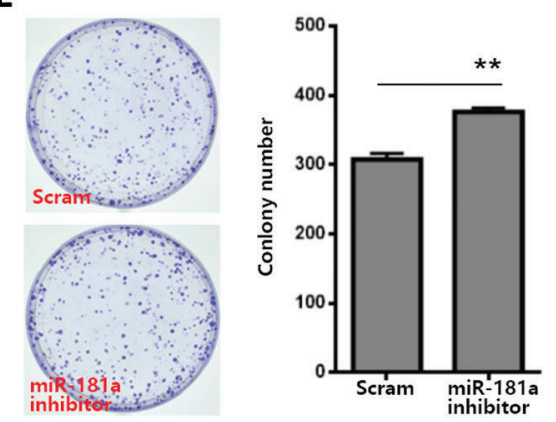

G
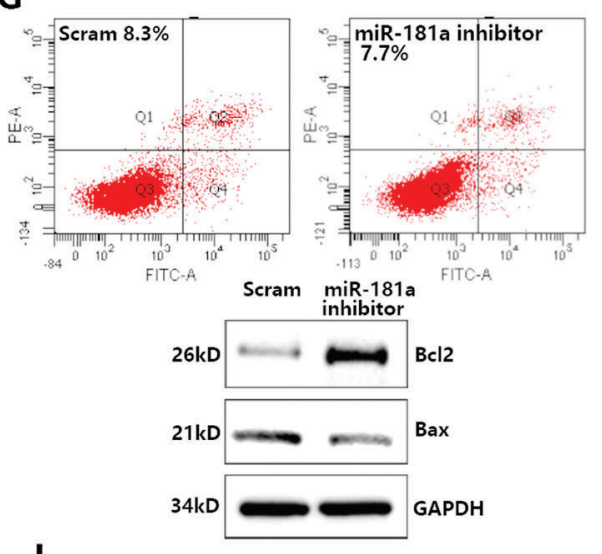

I
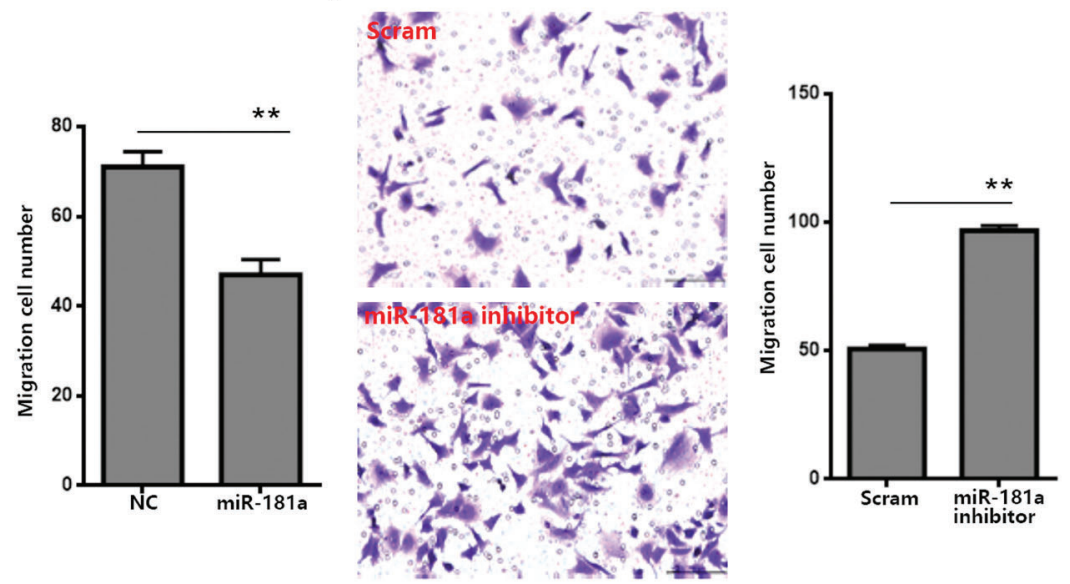

FIGURE 3. (continued) 
J

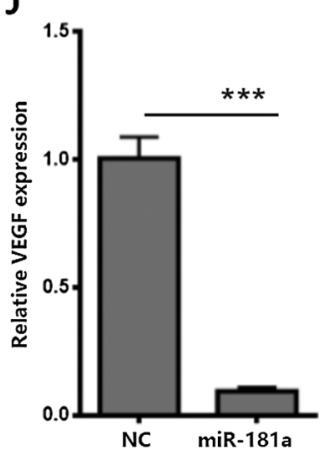

$\mathbf{L}$

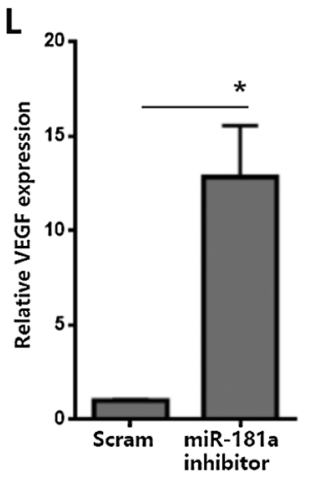

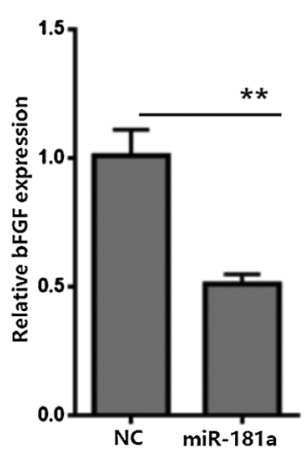

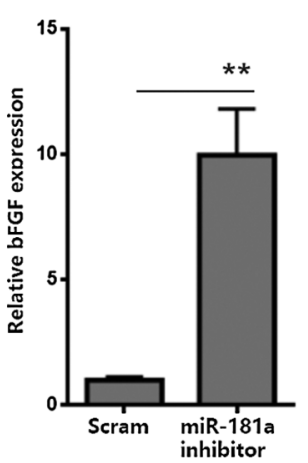

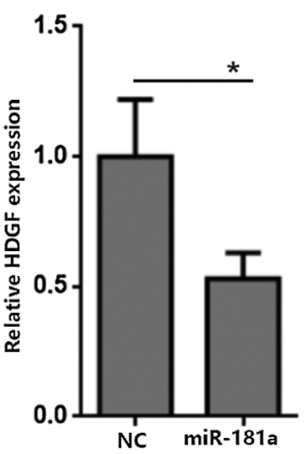
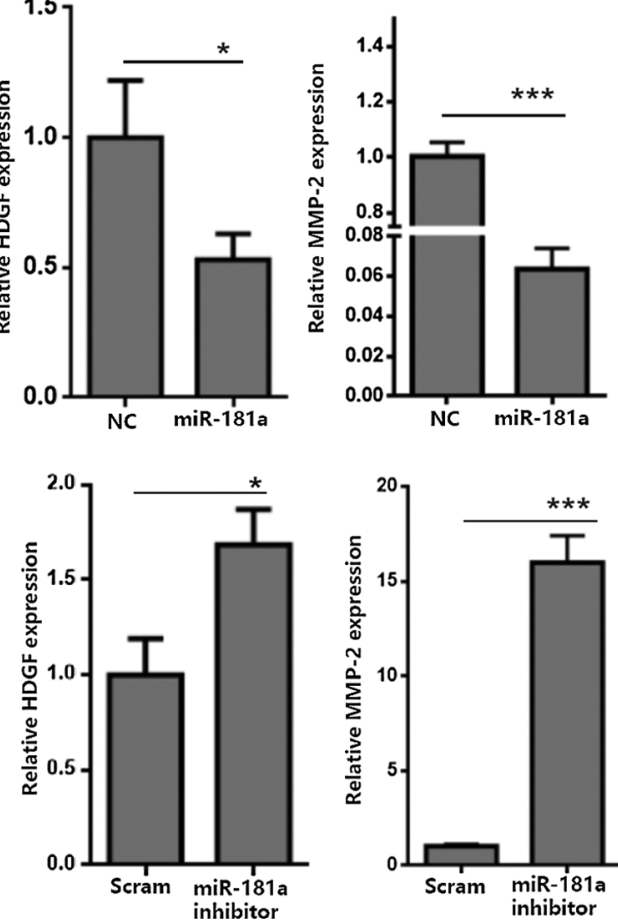

K

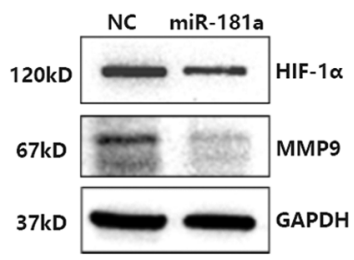

M
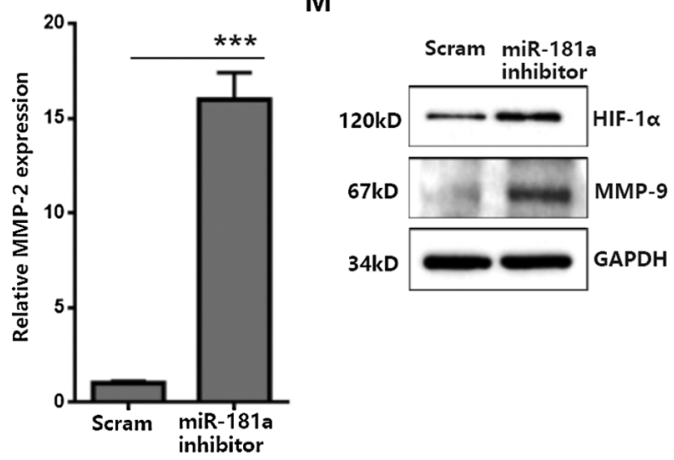

FIGURE 3. MiR-181a suppressed the proliferation and migration of cancer cells and promoted apoptosis.

(A) qRT-PCR analysis of miR-181a expression after siRNA transfection in A549 cells. Left panel: miR-181a vs. NC control; Right panel: miR181a inhibition $v s$. Scram control. (B-C) MTT assay of A549 at $48 \mathrm{~h}$ post-transfection of miRNAs. (B); miR-181a $v s$. NC control (C) miR-181a inhibition $v s$. Scram control. Data are expressed as mean \pm SD for triplicate experiments. ${ }^{\star \star} P<0.01$; ${ }^{\star *} P<0.001$; unpaired $t$-test. (D-E) Colony formation assays of A549 at $48 \mathrm{~h}$ post-transfection of miRNAs. (D) miR-181a vs. NC control; (E) miR-181a inhibition vs. Scram control. Data are expressed as mean \pm SD for triplicate experiments. ${ }^{\star *} P<0.01$; unpaired $t$-test. (F-G) FACS and western blot analysis of A549 cell apoptosis at $48 \mathrm{~h}$ post-transfection of miRNAs. (F) miR-181a vs. NC control; (G) miR-181a inhibition vs. Scram control. upper panel: FACS analysis of A549 cells; lower panel: western blot analysis of Bcl2 and Bax expression. (H-I) Transwell migration assay of A549 of $48 \mathrm{~h}$ post-transfection of miRNAs. (H) miR-181a vs. NC control; (I) miR-181a inhibition vs. Scram control. Data are expressed as mean \pm SD for triplicate experiments. ${ }^{* *} P<0.01$; unpaired $t$-test. (J, L) qRT-PCR analysis of VEGF, bFGF, HDGF, and MMP2 expression in A549 cells of $24 \mathrm{~h}$ post-transfection of miRNAs. (J) miR-181a vs. NC control; (L) miR-181a inhibition vs. Scram control. Data are expressed as mean \pm SD for triplicate experiments. ${ }^{*} P<0.01$; ${ }^{* *} P<0.001$; unpaired $t$-test. $(\mathrm{K}, \mathrm{M})$ Western blot analysis of HIF-1 $\alpha$ and MMP9 expression in lung carcinoma cells transfected with miRNAs. (K) miR-181a vs. NC control; (M) miR-181a inhibition vs. Scram control.

and angiogenesis (John and Tuszynski, 2001; Merchant et al., 2017). In light of the anti-angiogenic role of miR-181a in lung carcinoma cells, we also assessed neovascularization in xenografts using CD31 antibody, which only interacts with mouse endothelial cells. The results showed that miR-181a transfection in xenografts attenuated endothelial cell recruitment and the formation of novel vessels. Hence, miR181a not only plays an anti-tumor role in suppressing the proliferation and migration of lung carcinoma but also may regulate the expression of pro-angiogenic factors in tumor cells to modify the lung carcinoma microenvironment.

miRNAs decrease gene expression by binding to the 3'UTR of the target mRNAs, which leads to degradation or prevents translation. Our data indicated that miR-181a negatively regulates FOXP1 expression through binding to the 3'UTR of FOXP1 mRNA. FOXP1 is a member of the winged-helix transcription factors that are involved in a broad range of functions, such as cell cycle progression, proliferation, migration, and angiogenesis. FOXP1 functions as a cancer driver in some tumors but as a tumor suppressor in others (Katoh et al., 2013). Xu et al. (2020) reported that knockdown of FOXP1 promoted the development of lung adenocarcinoma (Sheng et al., 2019). In contrast, our results revealed that FOXP1 expression was elevated in lung carcinoma tissues, which was consistent with the results of a study by Feng et al. (2012). Although the precise role of FOXP1 in lung carcinoma remains to be elucidated, our results show that FOXP1 functions as an angiogenesis stimulator and promotes migration and proliferation of endothelial cells. Moreover, several studies found that FOXP1 activates the PI3K/Akt signaling pathways, which play pivotal roles in angiogenesis (He et al., 2018). Activation of the PI3K/Akt signaling pathway in carcinoma cells promoted the expression of HIF- $\alpha$, which upregulated the expression of VEGF and other angiogenic factors such as bFGF and MMPs (Tsubaki et al., 2019; Xu et al., 2020). In the present study, we found that miR-181a suppressed the expression of FOXP1 and deactivated PI3K/Akt signaling pathways. miR-181a transfection also attenuated FOXP1 expression in xenografts and subsequently decreased VEGF, bFGF, and MMP2 expressions. Our results also revealed that 10-HCPT may be a potential anti-angiogenic agent due to its capability of repressing neovascularization and angiogenic factor expression. More importantly, miR-181a expression was upregulated by $10-\mathrm{HCPT}$ so that miR-181a may partly mediate the process of FOXP1 upregulation and PI3K/Akt 
A

\begin{tabular}{|c|c|}
\hline & predicted pairing of target region (top) and miRNA (bottom) \\
\hline Position 687-712 of FOXP1 3' UTR & $5^{\prime}$...UUCUGUACCUUGUGAUGAAUGUU... \\
\hline hsa-miR-181a & UGAGUGGCUGUCGCAACUUACAA \\
\hline
\end{tabular}
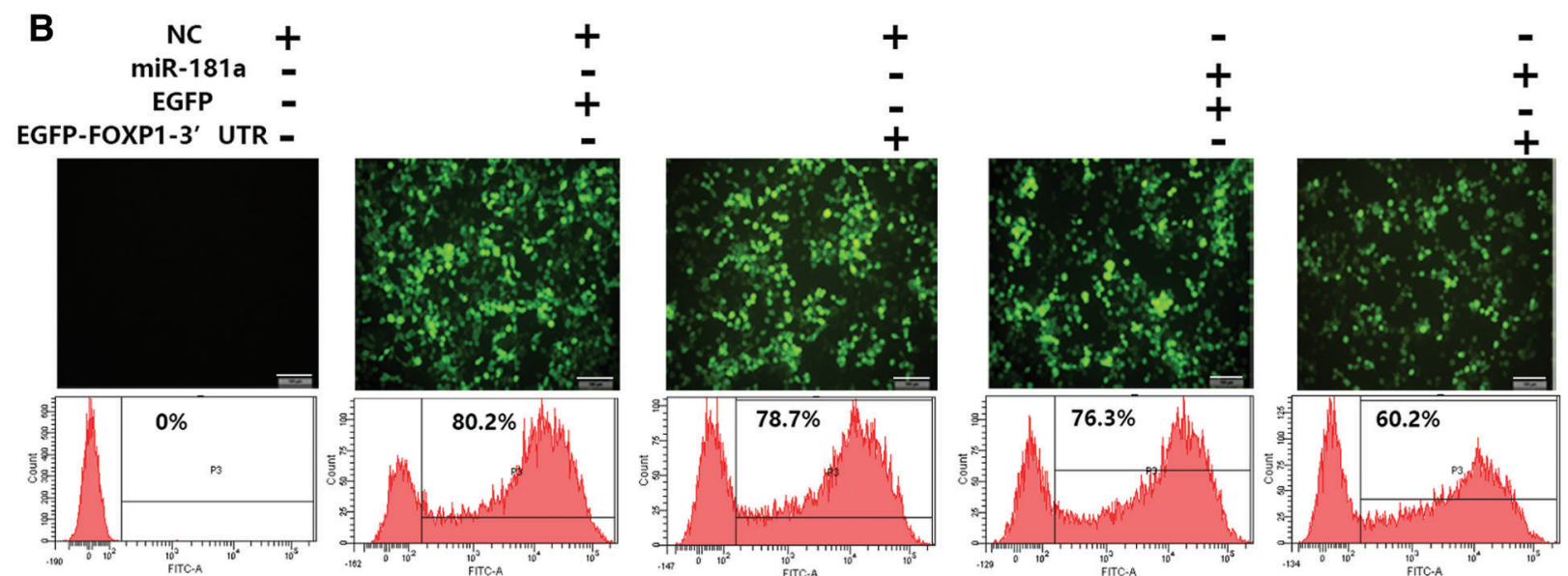

C

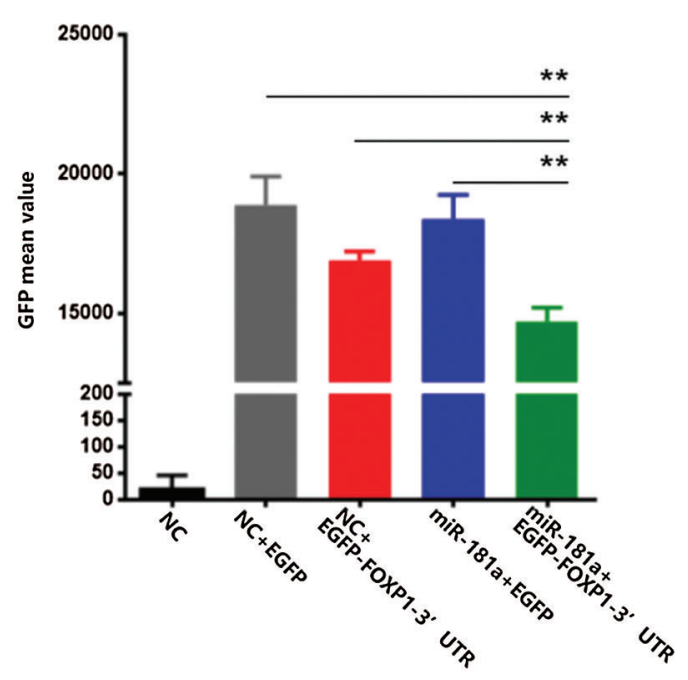

E
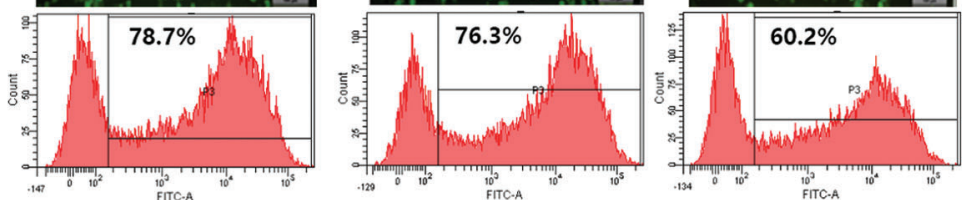

D
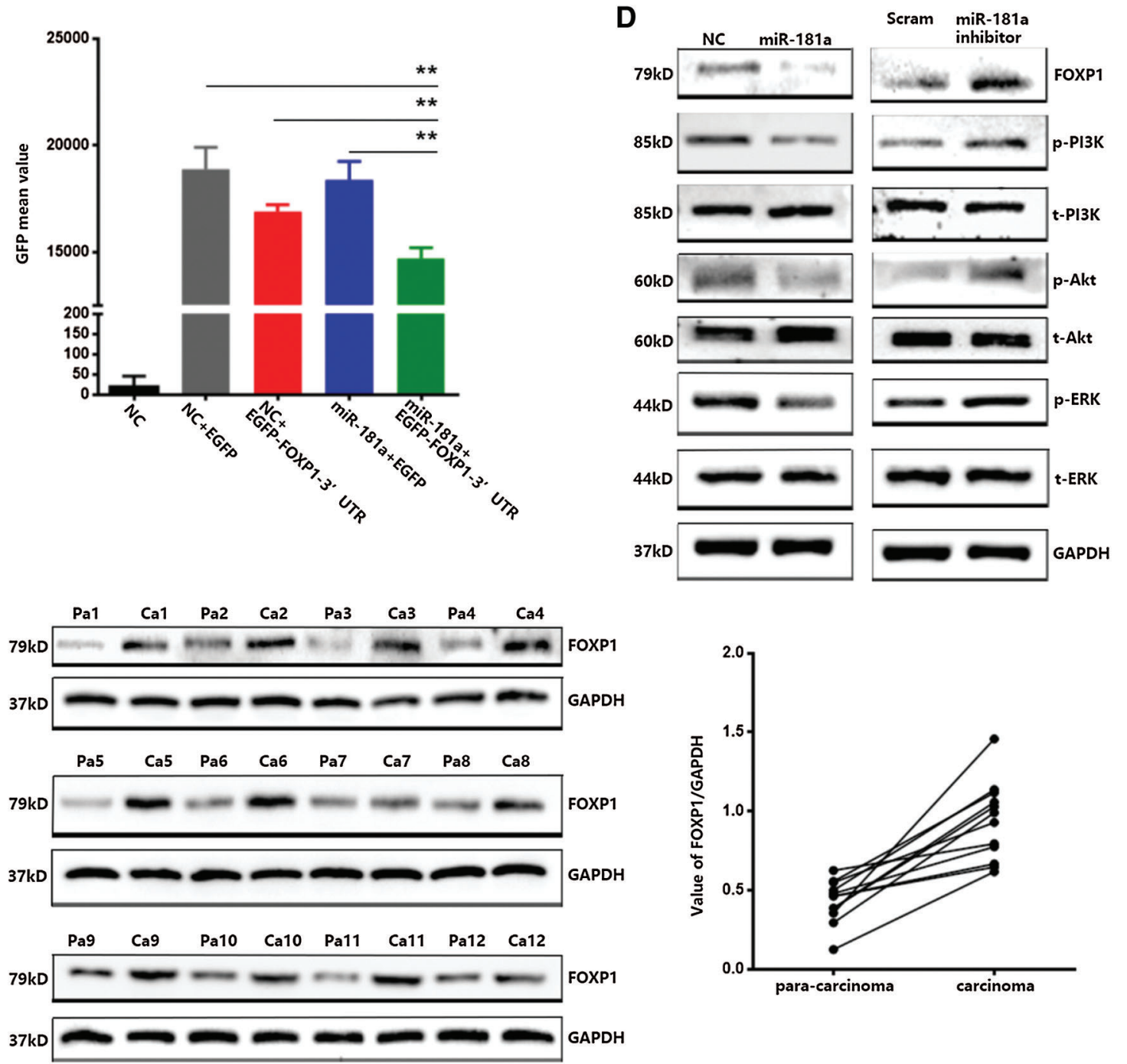

FIGURE 4. miR-181a targeted the 3'UTR of FOXP1, which was elevated in lung carcinoma tissue.

(A) TargetScan analysis predicted that the FOXP1 mRNA-3'-UTR is targeted by miR-181a. (B-C) FACS analysis of GFP expression in A549 cells co-transfected with miRNAs and GFP reported plasmids. (B) percentage of GFP positive cells; (C) GFP intensity. Data are expressed as mean \pm SD for triplicate experiments. (D) Western blot detection of FOXP1 expression and activation of PI3K/Akt/ERK pathway in lung carcinoma cells transfected with miRNAs. Left panel: miR-181a vs. NC control; right panel: miR-181a inhibition vs. Scram control. (E) Western blot analysis of FOXP1 expression in lung carcinoma tissue and its corresponding adjacent tissue. Right panel: statistical analysis of FOXP1 expression; carcinoma tissue $v$ s. para-carcinoma tissue, ${ }^{* * *} P<0.001$; paired $t$-test. 
A
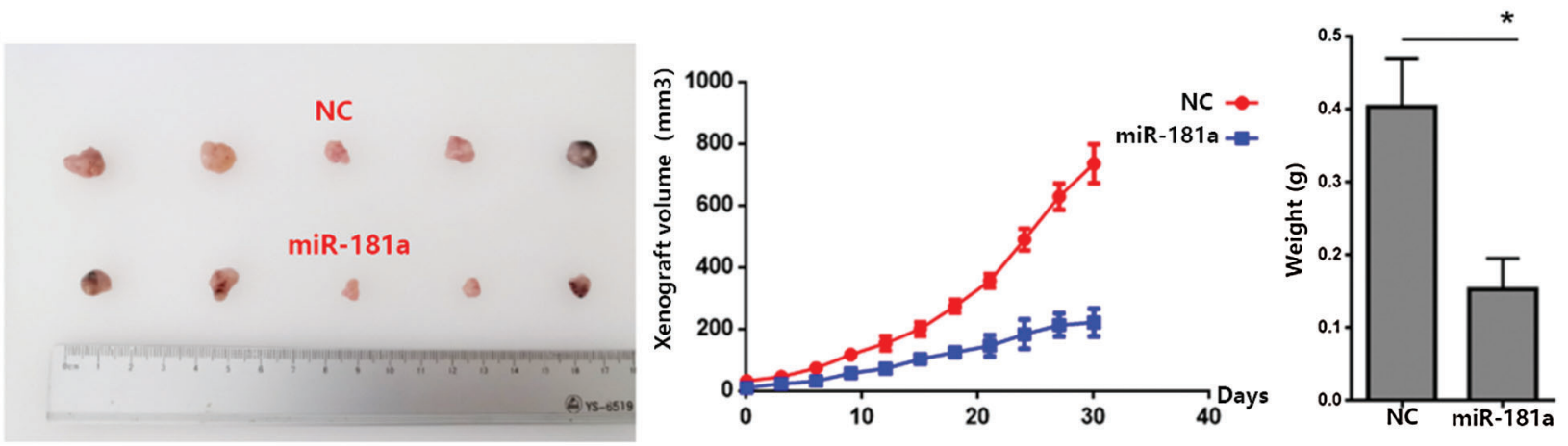

B
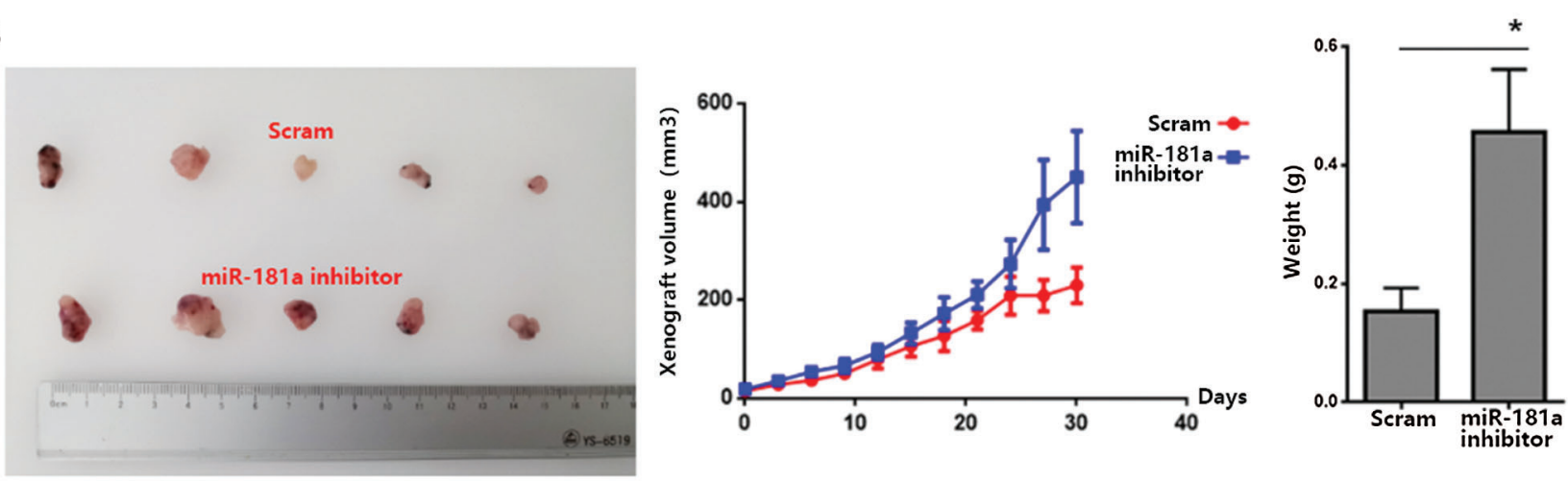

C

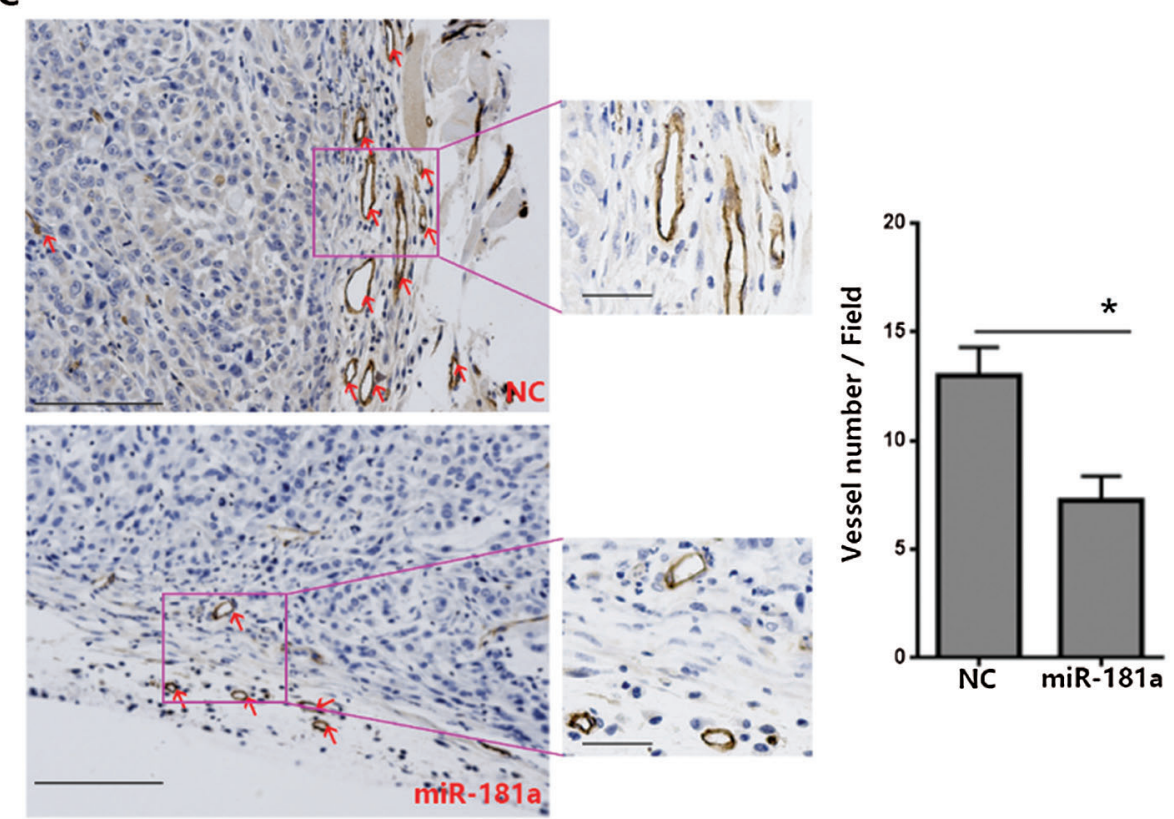

FIGURE 5. (continued) 
D
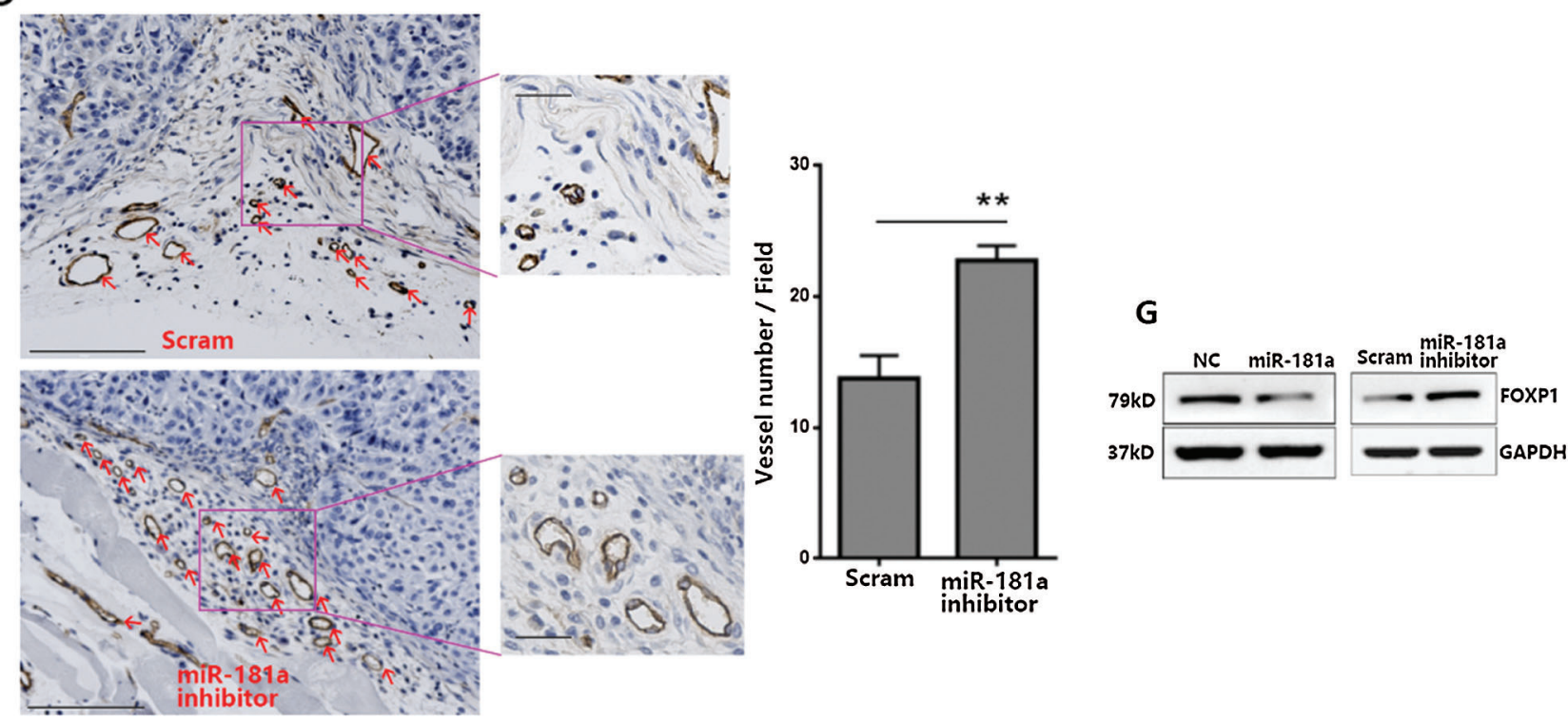

$\mathbf{E}$
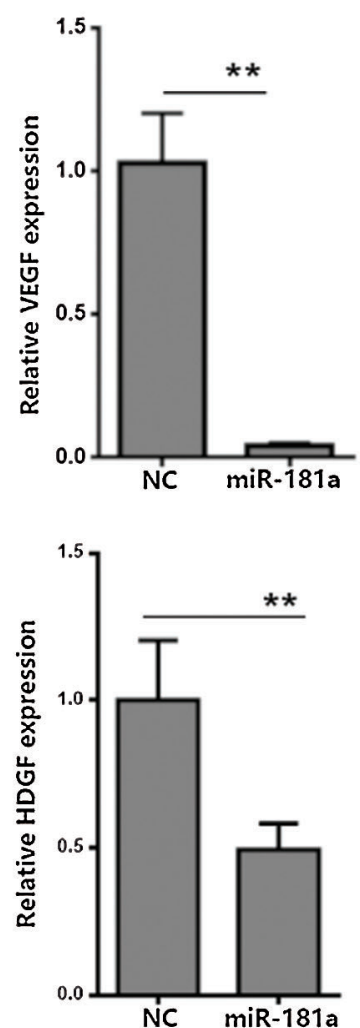
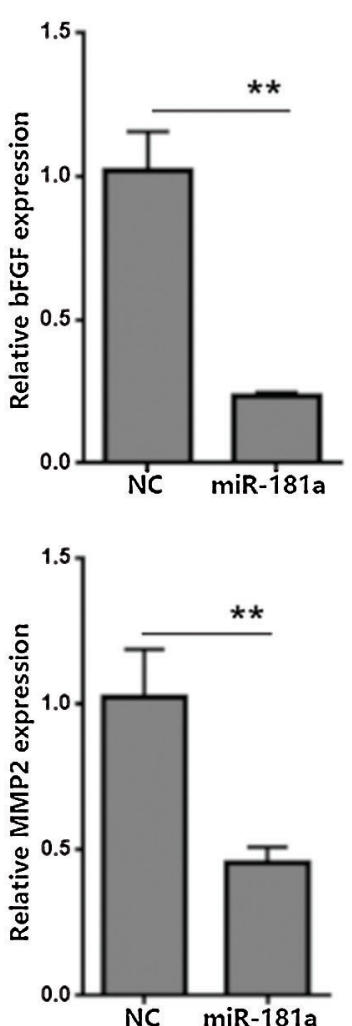

$\mathbf{F}$
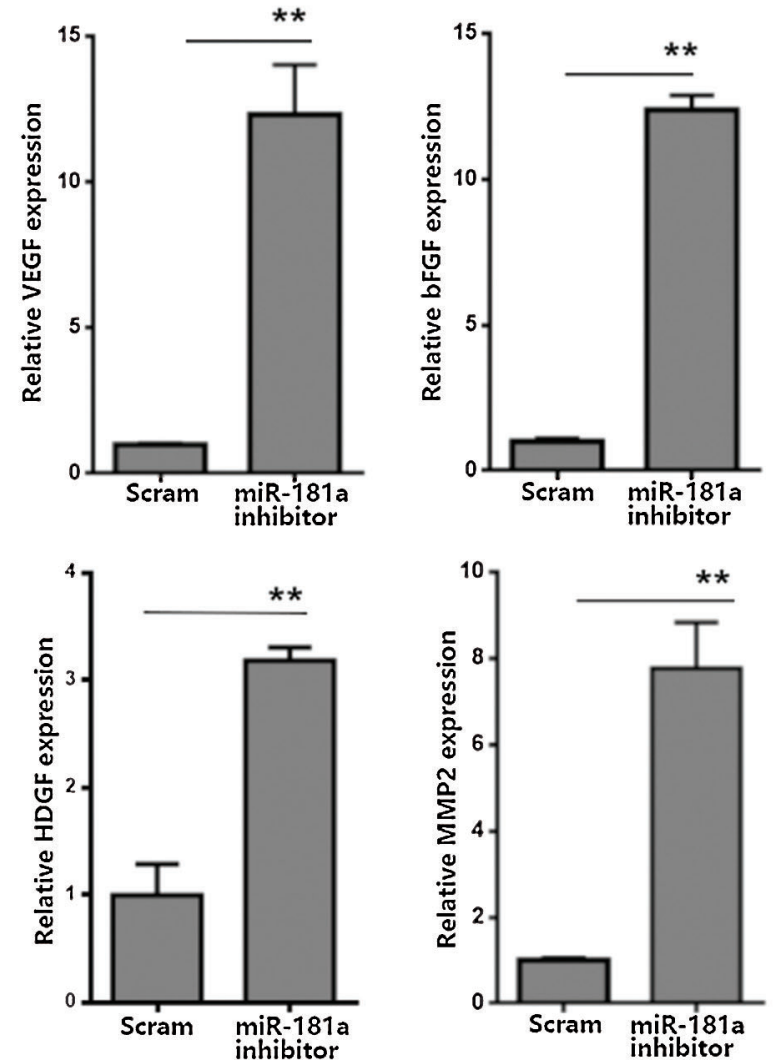

FIGURE 5. miR-181a suppressed tumorigenesis and angiogenesis in xenograft.

(A-B) Analysis of xenograft formed by A549 cells transfected with miRNAs (N = 5). Quantitative analysis of tumor volume (middle panel) and tumor weight (right panel) of xenografts. (A) miR-181a vs. NC control; (B) miR-181a inhibition $v s$. Scram control. Data are expressed as mean \pm SD. ${ }^{*} P<0.05$; Mann-Whitney U-test. (C-D) Representative images of CD31 immunohistochemical staining of xenograft formed by A549 cells transfected with miRNAs. The red arrows indicated novel blood vessels formed by endothelial cells in xenografts. The neovascularization part was magnified. Blood vessels were quantified on one field from each xenograft. (C) miR-181a vs. NC control; (D) miR-181a inhibition $v s$. Scram control. Data are expressed as mean \pm SD for triplicated fields. ${ }^{\star} P<0.05 ;{ }^{*} P<0.01$; unpaired $t$-test. (E-F) qRT-PCR analysis of VEGF, bFGF, HDGF, and MMP2 expression in xenograft formed by A549 cells transfected with miRNAs. (E) miR-181a vs. NC control; (F) miR-181a inhibition $v$ s. Scram control. Data are expressed as mean \pm SD for triplicate experiments. ${ }^{*} P<0.01$; unpaired $t$-test. (G) Western blot detection of FOXP1 expression in xenograft. Left panel: miR-181a vs. NC control; right panel: miR-181a inhibition $v s$. Scram control. 
pathways activation by $10-\mathrm{HCPT}$ treatment. Notably, miR181a has been reported to potentially target over 500 different mRNAs, which may exert differential or even opposing effects on tumor angiogenesis. Future studies should systemically study the targets of miR-181a to fully elucidate the mechanism and role of miR-181a in lung carcinoma.

\section{Conclusion}

We demonstrated that miR-181a was decreased in both lung carcinoma tissues and lung carcinoma cell lines and that miR181a had anti-oncogenic functions in lung carcinoma by suppressing proliferation and metastasis but promoting apoptosis. In addition, miR-181a attenuated tumor angiogenesis in xenograft. We also discovered that the angiogenic functions of miR-181a were likely through modification of the carcinoma microenvironment. FOXP1 is one target through which miR181a downregulated the expression of angiogenic factors in lung carcinoma cells. Moreover, 10-HCPT, a chemotherapy drug, elevated the expression of miR-181a but suppressed FOXP1 and its related PI3K/Akt signaling pathways. Thus, the miR-181aFOXP1-PI3K/Akt axis may have great potential as targets of anti-angiogenic drug development.

Acknowledgement: We thank Gabrielle White Wolf, Ph.D., from Liwen Bianji, Edanz Editing China (www.liwenbianji. $\mathrm{cn} / \mathrm{ac}$ ), for editing the English text of a draft of this manuscript.

Authors' Contributions: Conception and design: SYX, YFY. Acquisition of data (provided animals, acquired, and managed patients, provided facilities, etc.): LP. Analysis and interpretation of data (e.g., statistical analysis, biostatistics, computational analysis): YFY, LP, WTY. Writing, review, and/or revision of the manuscript: YFY, SYX. Administrative, technical, or material support (i.e., reporting or organizing data, constructing databases): LP, WTY, YFY, DML, YLZ, RRW, PYW, YJL, JXX. Study supervision: SYX.

Availability of Data and Materials: All data generated or analyzed during this study are included in this published article (and its supplementary information files).

Funding Statement: The present study was supported by the National Natural Science Foundation of China (Nos. 81702296, 81772281), the Shandong Science and Technology Committee (Nos. 2017GSF18124, ZR2019PC019, ZR2019MH022), the Health Commission of Shandong Province (Nos. 2017WS737, 2019KJK014), and the Shandong Province Taishan Scholar Project (No. ts201712067).

Conflicts of Interest: The authors declare that they have no conflicts of interest to report regarding the present study.

\section{References}

Cersosimo RJ (2002). Lung cancer: A review. American Journal of Health-System Pharmacy 59: 611-642. DOI 10.1093/ajhp/ 59.7.611.

Blandin Knight S, Crosbie PA, Balata H, Chudziak J, Hussell T, Dive C (2017). Progress and prospects of early detection in lung cancer. Open Biology 7: 170070. DOI 10.1098/rsob.170070.
Wani MC, Ronman PE, Lindley JT, Wall ME (1980). Plant antitumor agents. 18. Synthesis and biological activity of camptothecin analogues. Journal of Medicinal Chemistry 23: 554-560. DOI 10.1021/jm00179a016.

Ling YH, Andersson BS, Nelson JA (1990). DNA topoisomerase I as a site of action for 10-hydroxycamptothecin in human promyelocytic leukemia cells. Cancer Biochemistry Biophysics 11: 23-30.

Xiao D, Tan W, Li M, Ding J (2001). Antiangiogenic potential of 10-hydroxycamptothecin. Life Science 69: 1619-1628. DOI 10.1016/s0024-3205(01)01236-x.

Olgen S (2018). Overview on anticancer drug design and development. Current Medicinal Chemistry 25: 1704-1719. DOI 10.2174/0929867325666171129215610.

Vasudev NS, Reynolds AR (2014). Anti-angiogenic therapy for cancer: Current progress, unresolved questions and future directions. Angiogenesis 17: 471-494. DOI 10.1007/s10456014-9420-y.

Gacche RN, Meshram RJ (2014). Angiogenic factors as potential drug target: Efficacy and limitations of anti-angiogenic therapy. Biochimica et Biophysica Acta 1846: 161-179. DOI 10.1016/j.bbcan.2014.05.002.

Bergers G, Benjamin LE (2003). Tumorigenesis and the angiogenic switch. Nature Reviews Cancer 3: 401-410. DOI 10.1038/nrc1093.

Eguchi R, Wakabayashi I (2020). HDGF enhances VEGFdependent angiogenesis and FGF2 is a VEGFindependent angiogenic factor in nonsmall cell lung cancer. Oncology Reports 44: 14-28. DOI 10.3892/or.2020.7580.

Yang G, Yin B (2014). The advance of application for microRNAs in cancer gene therapy. Biomedicine \& Pharmacotherapy 68: 137-142. DOI 10.1016/j.biopha.2013.10.002.

Zhou Q, Huang SX, Zhang F, Li SJ, Liu C, Xi YY, Wang L, Wang X, He QQ, Sun CC, Li DJ (2017). MicroRNAs: A novel potential biomarker for diagnosis and therapy in patients with nonsmall cell lung cancer. Cell Proliferation 50: e12394. DOI 10.1111/cpr.12394.

Rezaei T, Amini M, Hashemi ZS, Mansoori B, Rezaei S, Karami H, Mosafer J, Mokhtarzadeh A, Baradaran B (2020). microRNA181 serves as a dual-role regulator in the development of human cancers. Free Radical Biology and Medicine 152: 432454. DOI 10.1016/j.freeradbiomed.2019.12.043.

Ji J, Yamashita T, Budhu A, Forgues M, Jia HL, Li C, Deng C, Wauthier E, Reid LM, Ye QH, Qin LX, Yang W, Wang HY, Tang ZY, Croce CM, Wang XW (2009). Identification of microRNA-181 by genome-wide screening as a critical player in EpCAM-positive hepatic cancer stem cells. Hepatology 50: 472-480. DOI 10.1002/hep.22989.

Wei Z, Cui L, Mei Z, Liu M, Zhang D (2014). miR-181a mediates metabolic shift in colon cancer cells via the PTEN/AKT pathway. FEBS Letters 588: 1773-1779. DOI 10.1016/j. febslet.2014.03.037.

Li Y, Kuscu C, Banach A, Zhang Q, Pulkoski-Gross A, Kim D, Liu J, Roth E, Li E, Shroyer KR, Denoya PI, Zhu X, Chen L, Cao J (2015). miR-181a-5p inhibits cancer cell migration and angiogenesis via downregulation of matrix metalloproteinase-14. Cancer Research 75: 2674-2685. DOI 10.1158/0008-5472.CAN-14-2875.

Ke Y, Zhao W, Xiong J, Cao R (2013). Downregulation of miR-16 promotes growth and motility by targeting HDGF in nonsmall cell lung cancer cells. FEBS Letters 587: 3153-3157. DOI 10.1016/j.febslet.2013.08.010.

Zhang YX, Yan YF, Liu YM, Li YJ, Zhang HH, Pang M, Hu JX, Zhao W, Xie N, Zhou L, Wang PY, Xie SY (2016). Smad3-related 
miRNAs regulated oncogenic TRIB2 promoter activity to effectively suppress lung adenocarcinoma growth. Cell Death \& Disease 7: e2528. DOI 10.1038/cddis.2016.432.

He Q, Zhao L, Liu Y, Liu X, Zheng J, Yu H, Cai H, Ma J, Liu L, Wang $\mathrm{P}$, Li Z, Xue Y (2018). circ-SHKBP1 regulates the angiogenesis of U87 glioma-exposed endothelial cells through miR-544a/FOXP1 and miR-379/FOXP2 pathways. Molecular Therapy-Nucleic Acids 10: 331-348. DOI 10.1016/j.omtn.2017.12.014.

Zeng L, Sun Y, Li X, Wang J, Yan L (2019). 10Hydroxycamptothecin induces apoptosis in human fibroblasts by regulating miRNA23b3p expression. Molecular Medicine Reports 19: 2680-2686. DOI 10.3892/mmr.2019.9927.

Wu XM, Shao XQ, Meng XX, Zhang XN, Zhu L, Liu SX, Lin J, Xiao HS (2011). Genome-wide analysis of microRNA and mRNA expression signatures in hydroxycamptothecin-resistant gastric cancer cells. Acta Pharmacologica Sinica 32: 259269. DOI 10.1038/aps.2010.204.

Zhao J, Nie Y, Wang H, Lin Y (2016). MiR-181a suppresses autophagy and sensitizes gastric cancer cells to cisplatin. Gene 576: 828-833. DOI 10.1016/j.gene.2015.11.013.

Armstrong CM, Liu C, Lou W, Lombard AP, Evans CP, Gao AC (2017). MicroRNA-181a promotes docetaxel resistance in prostate cancer cells. Prostate 77: 1020-1028. DOI 10.1002/ pros.23358.

Viallard C, Larrivee B (2017). Tumor angiogenesis and vascular normalization: Alternative therapeutic targets. Angiogenesis 20: 409-426. DOI 10.1007/s10456-017-9562-9.

Carmeliet P, Jain RK (2011). Molecular mechanisms and clinical applications of angiogenesis. Nature 473: 298-307. DOI 10.1038/nature10144.

Rahma OE, Hodi FS (2019). The Intersection between tumor angiogenesis and immune suppression. Clinical Cancer Research 25: 5449-5457. DOI 10.1158/1078-0432.CCR-18-1543.

Rupaimoole R, Calin GA, Lopez-Berestein G, Sood AK (2016). miRNA deregulation in cancer cells and the tumor microenvironment. Cancer Discovery 6: 235-246. DOI 10.1158/2159-8290.CD-15-0893.
Gao F, Liang B, Reddy ST, Farias-Eisner R, Su X (2014). Role of inflammation-associated microenvironment in tumorigenesis and metastasis. Current Cancer Drug Targets 14: 30-45. DOI 10.2174/15680096113136660107.

Bradshaw M, Mansfield A, Peikert T (2013). The role of vascular endothelial growth factor in the pathogenesis, diagnosis and treatment of malignant pleural effusion. Current Oncology Reports 15: 207-216. DOI 10.1007/s11912-013-0315-7.

Chen Y, Mathy NW, Lu H (2018). The role of VEGF in the diagnosis and treatment of malignant pleural effusion in patients with nonsmall cell lung cancer (Review). Molecular Medicine Reports 17: 8019-8030. DOI 10.3892/mmr.2018.8922.

John A, Tuszynski G (2001). The role of matrix metalloproteinases in tumor angiogenesis and tumor metastasis. Pathology \& Oncology Research 7: 14-23. DOI 10.1007/BF03032599.

Merchant N, Nagaraju GP, Rajitha B, Lammata S, Jella KK, Buchwald ZS, Lakka SS, Ali AN (2017). Matrix metalloproteinases: Their functional role in lung cancer. Carcinogenesis 38: 766-780. DOI 10.1093/carcin/bgx063.

Katoh M, Igarashi M, Fukuda H, Nakagama H, Katoh M (2013). Cancer genetics and genomics of human FOX family genes. Cancer Letters 328: 198-206. DOI 10.1016/j.canlet.2012.09.017.

Sheng H, Li X, Xu Y (2019). Knockdown of FOXP1 promotes the development of lung adenocarcinoma. Cancer Biology \& Therapy 20: 537-545. DOI 10.1080/15384047.2018.1537999.

Feng J, Zhang X, Zhu H, Wang X, Ni S, Huang J (2012). High expression of FoxP1 is associated with improved survival in patients with non-small cell lung cancer. American Journal of Clinical Pathology 138: 230-235. DOI 10.1309/ AJCPDHQFNYJZ01YG.

Tsubaki M, Takeda T, Tomonari Y, Koumoto YI, Imano M, Satou T, Nishida S (2019). Overexpression of HIF-1alpha contributes to melphalan resistance in multiple myeloma cells by activation of ERK1/2, Akt, and NF-kappaB. Laboratory Investigation 99: 72-84. DOI 10.1038/s41374-018-0114-8.

Xu F, Na L, Li Y, Chen L (2020). Roles of the PI3K/AKT/mTOR signalling pathways in neurodegenerative diseases and tumours. Cell Bioscience 10: 54. DOI 10.1186/s13578-020-00416-0. 


\section{Supplemental Material}

A
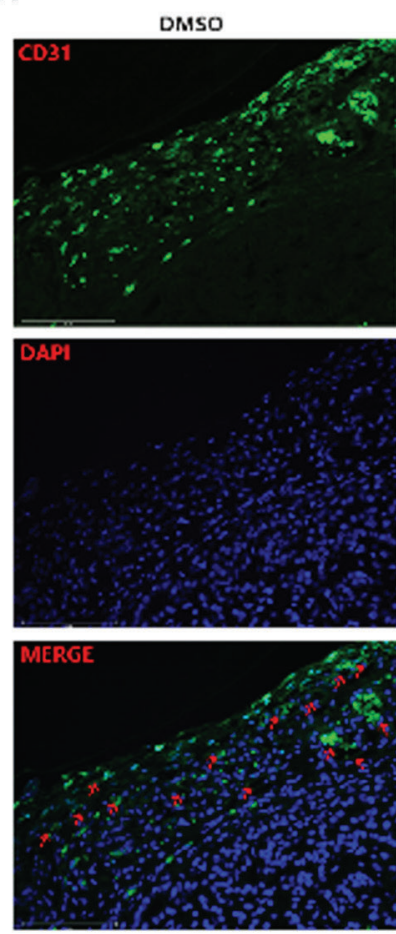

C
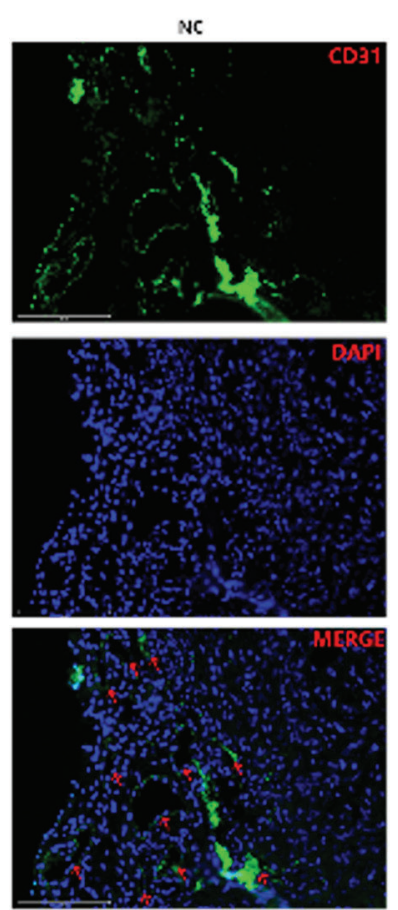

10.HCPT
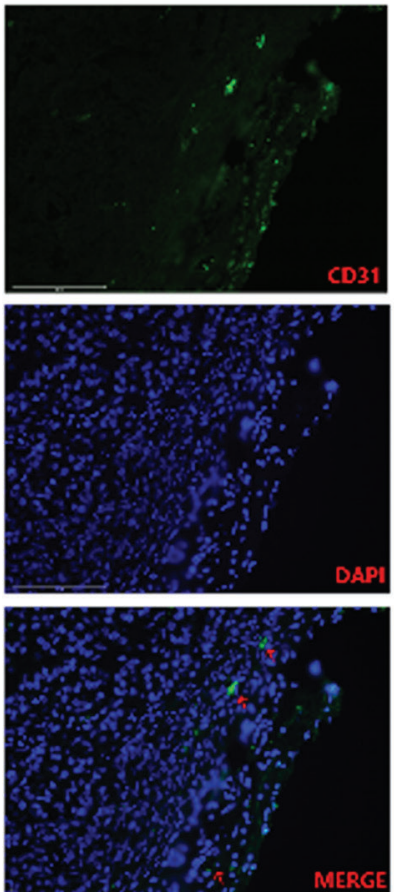

miR-181a
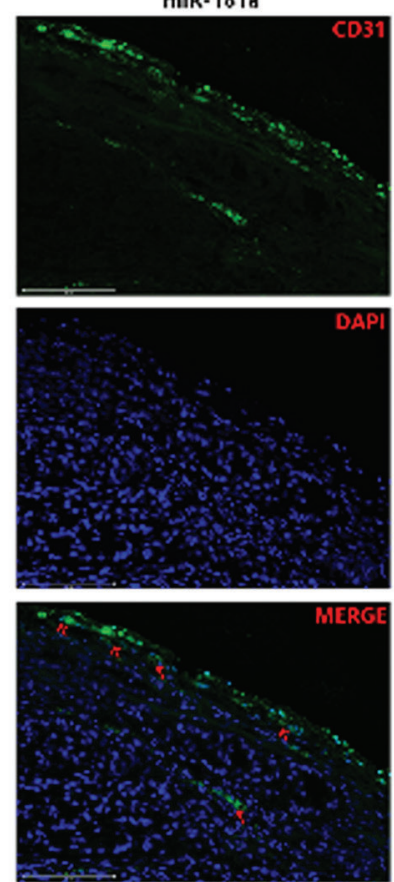

B
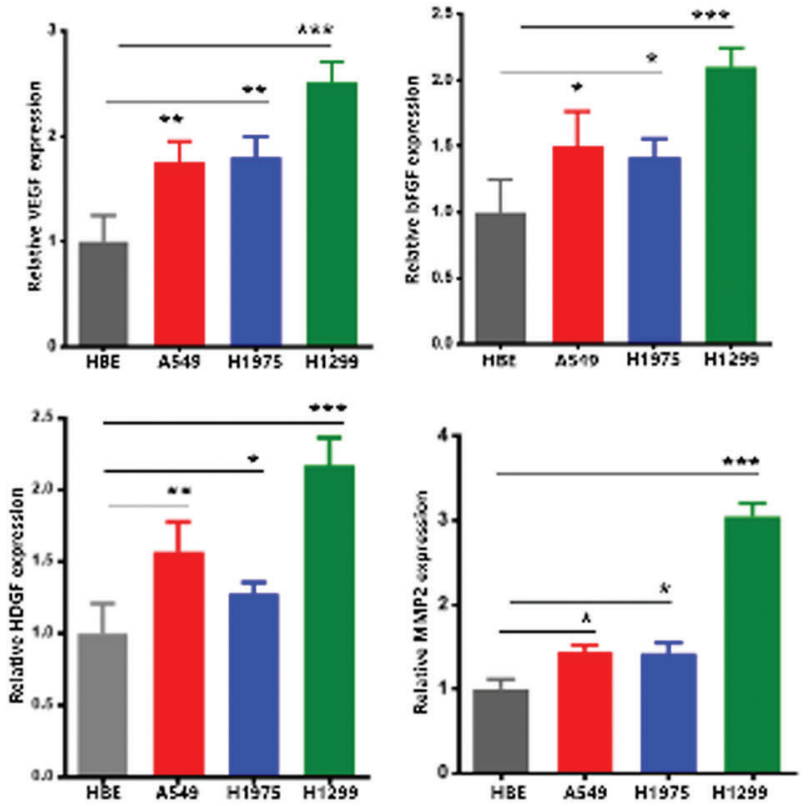

D
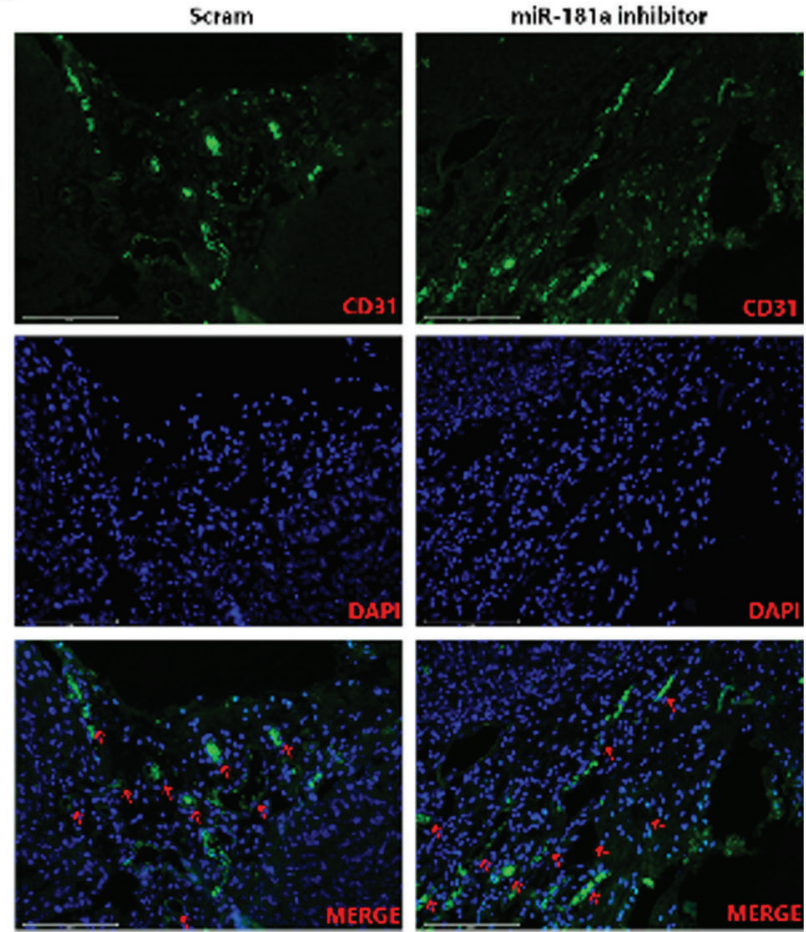

SUPPLEMENTARY FIGURE S1. (A) Representative images of CD31 fluorescent staining of xenograft formed by A549 cells treated with 10HCPT or DMSO. (B) qRT-PCR analysis of VEGF, bFGF, HDGF, and MMP2 expression in A549, H1975, H1299, and HBE cells. Data are expressed as mean \pm SD for triplicate experiments. ${ }^{* *} P<0.01$; ANOVA test. (C-D) Representative images of CD31 fluorescent staining of xenograft formed by A549 cells transfected with siRNAs 